1 Supplementary Material to Hui et al., Multi-Species Distribution 2 Modeling Using Penalized Mixture of Regressions

\title{
APPENDIX A: PROOFS
}

3 Proof of Theorem 1. We require the following regularity conditions on $\ell_{n}\left(\boldsymbol{\Psi}_{n}\right)$ :

4 A1. The density function $h_{n}\left(y ; \boldsymbol{x}, \mathbf{\Psi}_{n}\right)$ has common support on $(\boldsymbol{x}, y)$ and is identifiable in $\boldsymbol{\Psi}_{n}$ up to the permutation of the component labels.

6 A2. The Fisher information matrix

$$
\begin{aligned}
\mathcal{I}_{n}\left(\boldsymbol{\Psi}_{n}\right) & =E_{\mathbf{\Psi}_{n}^{0}}\left(\left[\frac{\partial \log h_{n}\left(y ; \boldsymbol{x}, \mathbf{\Psi}_{n}\right)}{\partial \boldsymbol{\Psi}_{n}}\right]\left[\frac{\partial \log h_{n}\left(y ; \boldsymbol{x}, \mathbf{\Psi}_{n}\right)}{\partial \mathbf{\Psi}_{n}}\right]^{\prime}\right) \\
& =E_{\boldsymbol{\Psi}_{n}^{0}}\left(-\frac{\partial^{2} \log h_{n}\left(y ; \boldsymbol{x}, \mathbf{\Psi}_{n}\right)}{\partial \mathbf{\Psi}_{n} \partial \mathbf{\Psi}_{n}^{\prime}}\right)
\end{aligned}
$$

is finite and positive definite at $\boldsymbol{\Psi}_{n}=\boldsymbol{\Psi}_{n}^{0}$.

A3. There exists an open subset $\omega_{n}$ of $\Omega_{n}$ containing the true parameters $\Psi_{n}^{0}$, such that for almost all $(\boldsymbol{x}, y)$ and any three elements $\Psi_{n, r}, \Psi_{n, s}$, and $\Psi_{n, t}$, there exists functions $M_{1}(\boldsymbol{x}, y)$ and $M_{2}(\boldsymbol{x}, y)$ with

$$
\left|\frac{\partial^{2} \log h_{n}\left(y ; \boldsymbol{x}, \mathbf{\Psi}_{n}\right)}{\partial \Psi_{n, r} \partial \Psi_{n, s}}\right|<M_{1, r s}(\boldsymbol{x}, y) ; \quad\left|\frac{\partial^{3} \log h_{n}\left(y ; \boldsymbol{x}, \mathbf{\Psi}_{n}\right)}{\partial \Psi_{n, r} \partial \Psi_{n, s} \partial \Psi_{n, t}}\right|<M_{2, r s t}(\boldsymbol{x}, y) .
$$

Moreover, $E_{\boldsymbol{\Psi}_{n}}\left(M_{1, r s}^{2}(\boldsymbol{x}, y)\right)<\infty$ and $E_{\boldsymbol{\Psi}_{n}}\left(M_{2, r s t}^{2}(\boldsymbol{x}, y)\right)<\infty$.

A4. For every $n$, there exists constants $\tau_{1}$ and $\tau_{2}$ such that

$$
0<\tau_{1}<\min \left\{\left(\beta_{n, k l}^{0}\right)^{2} ; \beta_{n, k l}^{0} \neq 0\right\}<\max \left\{\left(\beta_{n, k l}^{0}\right)^{2} ; \beta_{n, k l}^{0} \neq 0\right\}<\tau_{2}<\infty .
$$

Let $\alpha_{n}=\sqrt{p_{n} / n}$ and denote $\|\cdot\|$ as the $\ell_{2}$-norm. To prove estimation consistency, we show that for any $\varepsilon$ there exists a constant $C$ with $\|\boldsymbol{u}\|=C$ such that

$$
\lim _{n \rightarrow \infty} P\left(\sup _{\|\boldsymbol{u}\|=C} \ell_{n}^{\text {pen }}\left(\Psi_{n}^{0}+\alpha_{n} \boldsymbol{u}\right)<\ell_{n}^{\text {pen }}\left(\Psi_{n}^{0}\right)\right) \geq 1-\varepsilon
$$

15 where $\ell_{n}^{\text {pen }}\left(\boldsymbol{\Psi}_{n}^{0}\right)=\ell_{n}\left(\mathbf{\Psi}_{n}^{0}\right)-n \lambda_{n} \sum_{l=1}^{p_{n}} \tilde{w}_{n, l} \sqrt{\sum_{k=1}^{K}\left(\beta_{n, k l}^{0}\right)^{2}}$. This implies that with proba-

bility $1-\varepsilon$, there exists a local maximum inside the ball $\left\{\boldsymbol{\Psi}_{n}^{0}+\alpha_{n} \boldsymbol{u} ;\|\boldsymbol{u}\|=C\right\}$ and therefore $\left\|\hat{\mathbf{\Psi}}_{n}-\mathbf{\Psi}_{n}^{0}\right\|=O_{p}\left(\alpha_{n}\right)$. First, let

$$
\begin{aligned}
D_{n}(\boldsymbol{u}) & =\ell_{n}^{\text {pen }}\left(\boldsymbol{\Psi}_{n}^{0}+\alpha_{n} \boldsymbol{u}\right)-\ell_{n}^{\text {pen }}\left(\mathbf{\Psi}_{n}^{0}\right) \\
& \leq\left(\ell_{n}\left(\boldsymbol{\Psi}_{n}^{0}+\alpha_{n} \boldsymbol{u}\right)-\ell_{n}\left(\boldsymbol{\Psi}_{n}^{0}\right)\right)
\end{aligned}
$$




$$
\begin{aligned}
& \quad-\sum_{l \in \mathcal{C}_{n}^{c}} n \lambda_{n} \tilde{w}_{n, l}\left(\sqrt{\sum_{k=1}^{K}\left(\beta_{n, k l}^{0}+\alpha_{n} u_{k}\right)^{2}}-\sqrt{\sum_{k=1}^{K}\left(\beta_{n, k l}^{0}\right)^{2}}\right) \\
& \triangleq T_{n, 1}(\boldsymbol{u})-T_{n, 2}(\boldsymbol{u}) .
\end{aligned}
$$

To clarify, $l \in \mathcal{C}_{n}^{c}$ defines the set of covariates with at least one truly non-zero coefficient across the $K$ components, i.e. the set of partly and completely uninformative covariates. Applying a Taylor expansion, we have

$$
\begin{aligned}
T_{n, 1}(\boldsymbol{u}) & =\alpha_{n} \boldsymbol{u}^{\prime} \nabla \ell_{n}\left(\mathbf{\Psi}_{n}^{0}\right)+\frac{1}{2} \alpha_{n}^{2} \boldsymbol{u}^{\prime} \nabla^{2} \ell_{n}\left(\Psi_{n}^{0}\right) \boldsymbol{u}+\frac{1}{6} \alpha_{n}^{3} \boldsymbol{u}^{\prime} \nabla\left(\boldsymbol{u}^{\prime} \nabla^{2} \ell_{n}\left(\Psi_{n}^{*}\right) \boldsymbol{u}\right) \\
& \triangleq I_{1}+I_{2}+I_{3},
\end{aligned}
$$

$$
\left\|\frac{1}{n} \nabla^{2} \ell_{n}\left(\mathbf{\Psi}_{n}^{0}\right)+\mathcal{I}_{n}\left(\mathbf{\Psi}_{n}^{0}\right)\right\|=o_{p}\left(p_{n}^{-1}\right) .
$$

Lastly, by the Cauchy-Schwarz inequality we have

$$
\left|\boldsymbol{u}^{\prime} \nabla\left(\boldsymbol{u}^{\prime} \nabla^{2} \ell_{n}\left(\Psi_{n}^{*}\right) \boldsymbol{u}\right)\right| \leq\|\boldsymbol{u}\|^{3} \sum_{i=1}^{n}\left(\sum_{r, s, t} M_{n, 2, r s t}^{2}(\boldsymbol{x}, y)\right)^{1 / 2}=O_{p}\left(p_{n}^{3 / 2} n\right)\|\boldsymbol{u}\|^{3} .
$$

$$
\left|I_{1}\right|=o_{p}\left(n \alpha_{n}^{2}\right)\|\boldsymbol{u}\| ; \quad I_{2}=-\frac{1}{2} n \alpha_{n}^{2} \boldsymbol{u}^{\prime} \mathcal{I}_{n}\left(\Psi_{n}^{0}\right) \boldsymbol{u}\left(1+o_{p}(1)\right) ; \quad\left|I_{3}\right|=o_{p}\left(n \alpha_{n}^{2}\right)\|\boldsymbol{u}\|^{3},
$$

25 where the order of $\left|I_{3}\right|$ follows from condition (C).

26 Turning to $T_{n, 2}(\boldsymbol{u})$, observe that $\left(\beta_{n, k l}^{0}+\alpha_{n} u_{k}\right)^{2} \geq\left(\beta_{n, k l}^{0}\right)^{2}-2 \alpha_{n}\left|u_{k} \beta_{n, k l}^{0}\right| \geq 0$ for $n$ 27 large enough. Thus for $l \in \mathcal{C}_{n}^{c}$, we have

$$
\begin{aligned}
& \sqrt{\sum_{k=1}^{K}\left(\beta_{n, k l}^{0}+\alpha_{n} u_{k}\right)^{2}}-\sqrt{\sum_{k=1}^{K}\left(\beta_{n, k l}^{0}\right)^{2}} \\
& \geq \sqrt{\sum_{k=1}^{K}\left(\left(\beta_{n, k l}^{0}\right)^{2}-2 \alpha_{n} \mid u_{k} \beta_{n, k l}^{0}\right)}-\sqrt{\sum_{k=1}^{K}\left(\beta_{n, k l}^{0}\right)^{2}} \\
& \geq \theta_{n}^{0}\left(\sqrt{1-\delta_{n}^{0}+O\left(\left(\delta_{n}^{0}\right)^{2}\right)}-1\right)
\end{aligned}
$$




$$
\left.\frac{\partial \ell_{n}\left(\mathbf{\Psi}_{n}\right)}{\partial \beta_{n, k l}}\right|_{\hat{\mathbf{\Psi}}_{n}}=\operatorname{sgn}\left(\hat{\beta}_{n, k l}\right) n \lambda_{n} \tilde{w}_{n, l} \mid \hat{\beta}_{n, k l}\left(\sum_{k=1}^{K} \hat{\beta}_{n, k l}^{2}\right)^{-1 / 2}
$$

where $\theta_{n}^{0}=\sqrt{\sum_{k=1}^{K}\left(\beta_{n, k l}^{0}\right)^{2}}$ and $\delta_{n}^{0}=\alpha_{n} \sum_{k=1}^{K}\left(2\left|u_{k} \beta_{n, k l}^{0}\right|-u_{k}\left(\beta_{n, k l}^{0}\right)^{2}\right) /\left(\theta_{n}^{0}\right)^{2} \stackrel{p}{\rightarrow} 0$. Applying the Taylor expansion $\sqrt{1-\delta_{n}^{0}+O\left(\left(\delta_{n}^{0}\right)^{2}\right)}=1-(1 / 2) \delta_{n}^{0}+O\left(\left(\delta_{n}^{0}\right)^{2}\right)$ for $\delta_{n} \rightarrow 0$, we find that the absolute value of the $l^{\text {th }}$ summand in $-T_{n, 2}(\boldsymbol{u})$ is bounded by,

$$
\begin{aligned}
\left|-T_{n, 2}(\boldsymbol{u})_{l}\right| & \leq\left|\frac{n \lambda_{n} \alpha_{n} \tilde{w}_{n, l} \sum_{k=1}^{K}\left(\left|u_{k} \beta_{n, k l}^{0}\right|-0.5 u_{k}\left(\beta_{n, k l}^{0}\right)^{2}\right)}{\theta_{n}^{0}}\right|\left(1+\left|o_{p}(1)\right|\right) \\
& \leq\left|\frac{n \lambda_{n} \alpha_{n} a_{n} \sqrt{K}\left(\sqrt{\tau_{2}}+0.5 \tau_{2}\right)\|\boldsymbol{u}\|}{\sqrt{\tau_{1}}}\right|\left(1+\left|o_{p}(1)\right|\right),
\end{aligned}
$$

From condition (A), it follows that $n \lambda_{n} \alpha_{n} a_{n}=o_{p}\left(n \alpha_{n}^{2}\right)$ and hence $-T_{n, 2}(\boldsymbol{u})$ is bounded above by $o_{p}\left(n \alpha_{n}^{2}\right)\left(1+\left|o_{p}(1)\right|\right)$ uniformly for $\|\boldsymbol{u}\|=C$. Based on this result and those of (4), we observe that for $C$ large enough, $I_{2}$ dominates $I_{1}, I_{3}$ and $-T_{n, 2}(\boldsymbol{u})$. Since $I_{2}$ is negative, the required result in (1) and hence estimation consistency follows.

We now prove the second part of the theorem, namely covariate selection consistency. Suppose for a covariate $l \in \mathcal{C}_{n}$ we have $\left\|\hat{\boldsymbol{\beta}}_{n, l}\right\|_{2} \neq 0$. This implies that for all $k=$ $1, \ldots, K$,

where $\operatorname{sgn}(\cdot)$ is the sign function. We will show that with probability tending to 1 , the equality in (5) can not be satisfied. By the first part of Theorem 1 we know $\hat{\boldsymbol{\beta}}_{n}$ is a $\left(\sqrt{n / p_{n}}\right)$-consistent estimator, which implies $\left(n p_{n}\right)^{-1 / 2} \partial \ell_{n}\left(\boldsymbol{\Psi}_{n}\right) /\left.\partial \beta_{n, k l}\right|_{\hat{\mathbf{\Psi}}_{n}}=O_{p}(1)$. On the other hand, we have

$$
\begin{aligned}
\left(n p_{n}\right)^{-1 / 2} n \lambda_{n} \tilde{w}_{n, l}\left|\hat{\beta}_{n, k l}\right|\left(\sum_{k=1}^{K} \hat{\beta}_{n, k l}^{2}\right)^{-1 / 2} & \geq \sqrt{n / p_{n}} \lambda_{n} \sqrt{b_{n}}\left|\hat{\beta}_{n, k l}\right|\left(\sum_{k=1}^{K} \hat{\beta}_{n, k l}^{2}\right)^{-1 / 2} \\
& \geq O_{p}\left(\lambda_{n} \sqrt{n b_{n} / p_{n}}\right) .
\end{aligned}
$$

By condition (B), $\lambda_{n} \sqrt{n b_{n} / p_{n}} \rightarrow \infty$. Hence we conclude that the left hand side of (5) can not equal the right side with probability tending to 1 . It follows that $\hat{\boldsymbol{\beta}}_{n, k l}=0 \forall k=1, \ldots, K$, which implies $\left\|\hat{\boldsymbol{\beta}}_{n, l}\right\|_{2}=0$ and $\hat{\boldsymbol{\beta}}_{n, \mathcal{C}_{n}}=\mathbf{0}$ with probability tending to 1.

Finally, we prove the asymptotic normality property. Recall that $\mathcal{C}_{n}^{c}$ is the set of partly and completely uninformative covariates. Writing $\hat{\mathbf{\Psi}}_{n}=\left(\hat{\mathbf{\Psi}}_{n, \mathcal{C}_{n}^{c}}, \mathbf{0}\right)$ where 


$$
\begin{aligned}
& \frac{1}{n} \nabla \ell_{n}^{\text {pen }}\left(\hat{\mathbf{\Psi}}_{n, \mathcal{C}_{n}^{c}}\right)=\frac{1}{n} \nabla \ell_{n}^{\text {pen }}\left(\mathbf{\Psi}_{n, \mathcal{C}_{n}^{c}}^{0}\right)+\frac{1}{n} \nabla^{2} \ell_{n}^{\text {pen }}\left(\Psi_{n, \mathcal{C}_{n}^{c}}^{0}\right)\left(\hat{\mathbf{\Psi}}_{n, \mathcal{C}_{n}^{c}}-\Psi_{n, \mathcal{C}_{n}^{c}}^{0}\right) \\
& +\frac{1}{2 n}\left(\hat{\mathbf{\Psi}}_{n, \mathcal{C}_{n}^{c}}-\mathbf{\Psi}_{n, \mathcal{C}_{n}^{c}}^{0}\right)^{\prime} \nabla^{3} \ell_{n}^{\text {pen }}\left(\boldsymbol{\Psi}_{n, \mathcal{C}_{n}^{c}}^{*}\right)\left(\hat{\mathbf{\Psi}}_{n, \mathcal{C}_{n}^{c}}-\mathbf{\Psi}_{n, \mathcal{C}_{n}^{c}}^{0}\right) \\
& =\mathbf{0}
\end{aligned}
$$

where $\boldsymbol{\Psi}_{n, \mathcal{C}_{n}^{c}}^{*}$ lies on the line segment connecting $\hat{\mathbf{\Psi}}_{n, \mathcal{C}_{n}^{c}}$ and $\boldsymbol{\Psi}_{n, \mathcal{C}_{n}^{c}}^{0}$. To simplify (6), we utilize the following results. First, from the Cauchy-Schwarz inequality we obtain

$$
\begin{aligned}
\| \frac{1}{n}\left(\hat{\boldsymbol{\Psi}}_{n, \mathcal{C}_{n}^{c}}-\boldsymbol{\Psi}_{n, \mathcal{C}_{n}^{c}}^{0}\right)^{\prime} \nabla^{3} \ell_{n}\left(\boldsymbol{\Psi}_{n, \mathcal{C}_{n}^{c}}^{*}\right) & \left(\hat{\boldsymbol{\Psi}}_{n, \mathcal{C}_{n}^{c}}-\boldsymbol{\Psi}_{n, \mathcal{C}_{n}^{c}}^{0}\right) \| \\
& \leq \frac{1}{n}\left\|\left(\hat{\boldsymbol{\Psi}}_{n, \mathcal{C}_{n}^{c}}-\boldsymbol{\Psi}_{n, \mathcal{C}_{n}^{c}}^{0}\right)\right\|^{2} \sum_{i=1}^{n}\left(\sum_{r, s, t} M_{n, 2, r, s, t}^{2}(\boldsymbol{x}, y)\right)^{1 / 2} \\
& \leq \frac{1}{n} O_{p}\left(p_{n} / n\right) O_{p}\left(p_{n}^{3 / 2} n\right)=o_{p}\left(n^{-1 / 2}\right),
\end{aligned}
$$

where the last line of the above follows from condition $\left(\mathrm{C}^{\prime}\right)$. Next, denote $p\left(\boldsymbol{\beta}_{n, l}\right)=$ $\tilde{w}_{n, l}\left(\sum_{k=1}^{K} \beta_{n, k l}^{2}\right)^{1 / 2}$. Based on (5), we have for $l \in \mathcal{C}_{n}^{c}$,

$\left|\frac{1}{n} n \lambda_{n} \frac{\partial p\left(\boldsymbol{\beta}_{n, l}\right)}{\partial \beta_{n, k l}}\right|_{\boldsymbol{\Psi}_{n}^{0}}|=| \frac{\lambda_{n} \tilde{w}_{n, l} \beta_{n, k l}^{0}}{p\left(\boldsymbol{\beta}_{n, l}^{0}\right)}|<| \frac{\lambda_{n} a_{n} \beta_{n, k l}^{0}}{p\left(\boldsymbol{\beta}_{n, l}^{0}\right)} \mid=o_{p}\left(n^{-1 / 2} p_{n}^{-1}\right)=o_{p}\left(\left(n p_{n}\right)^{-1 / 2}\right)$.

under condition $\left(\mathrm{A}^{\prime}\right)$. From this result, it follows that $\left\|(1 / n) \nabla p\left(\boldsymbol{\Psi}_{n, \mathcal{C}_{n}^{c}}^{0}\right)\right\|=o_{p}\left(n^{-1 / 2}\right)$.

Similarly, it may be shown that $\left\|(1 / n) \nabla^{2} p\left(\mathbf{\Psi}_{n, \mathcal{C}_{n}^{c}}^{0}\right)\left(\hat{\Psi}_{n, \mathcal{C}_{n}^{c}}-\Psi_{n, \mathcal{C}_{n}^{c}}^{0}\right)\right\|=o_{p}\left(n^{-1 / 2}\right)$ and $\left\|(1 / n)\left(\hat{\mathbf{\Psi}}_{n, \mathcal{C}_{n}^{c}}-\boldsymbol{\Psi}_{n, \mathcal{C}_{n}^{c}}^{0}\right)^{\prime} \nabla^{3} p\left(\boldsymbol{\Psi}_{n, \mathcal{C}_{n}^{c}}^{*}\right)\left(\hat{\mathbf{\Psi}}_{n, \mathcal{C}_{n}^{c}}-\mathbf{\Psi}_{n, \mathcal{C}_{n}^{c}}^{0}\right)\right\|=o_{p}\left(n^{-1 / 2}\right)$. By utilizing these results, we can rearrange (6) to obtain

$$
\begin{aligned}
\frac{1}{n} \nabla \ell_{n}\left(\Psi_{n, \mathcal{C}_{n}^{c}}^{0}\right) & =-\frac{1}{n} \nabla^{2} \ell_{n}\left(\Psi_{n, \mathcal{C}_{n}^{c}}^{0}\right)\left(\hat{\Psi}_{n, \mathcal{C}_{n}^{c}}-\Psi_{n, \mathcal{C}_{n}^{c}}^{0}\right)+o_{p}\left(\frac{1}{\sqrt{n}}\right) \\
& =\mathcal{I}_{n}\left(\Psi_{n, \mathcal{C}_{n}^{c}}^{0}\right)\left(\hat{\Psi}_{n, \mathcal{C}_{n}^{c}}-\Psi_{n, \mathcal{C}_{n}^{c}}^{0}\right)+o_{p}\left(\frac{1}{\sqrt{n}}\right),
\end{aligned}
$$

where (7) follows from applying (3). From here, we adopt the same argument as in the proof of Theorem 2 in Fan and Peng (2004), using the central limit theorem (with 
the Lindeberg condition satisfied) to show that $n^{-1 / 2} \boldsymbol{\Gamma}_{n} \mathcal{I}_{n}\left(\Psi_{n, \mathcal{C}_{n}^{c}}^{0}\right)^{-1 / 2} \nabla \ell_{n}\left(\Psi_{n, \mathcal{C}_{n}^{c}}^{0}\right) \stackrel{p}{\rightarrow}$ $N(\mathbf{0}, \boldsymbol{G})$. Applying this result to (7) leads to the normality property as announced. Finally, it is clear that replacing $K$ in the above by some consistent estimator $\hat{K} \rightarrow$ $p K$ does not affect the proof.

Proof of Theorem 2. The proof is largely similar to the proof of Theorem 1 provided above, and is also very similar to the proof of the oracle property for the hierarchical LASSO (Zhou and Zhu, 2007). Therefore, we shall only provide a sketch derivation.

To demonstrate estimation consistency, we set about proving that equation (1) also holds true for the MIXGL1 penalty. We have

$$
\begin{aligned}
D_{n}(\boldsymbol{u}) & =\ell_{n}^{\text {pen }}\left(\boldsymbol{\Psi}_{n}^{0}+\alpha_{n} \boldsymbol{u}\right)-\ell_{n}^{\text {pen }}\left(\mathbf{\Psi}_{n}^{0}\right) \\
\leq & \left(\ell_{n}\left(\mathbf{\Psi}_{n}^{0}+\alpha_{n} \boldsymbol{u}\right)-\ell_{n}\left(\mathbf{\Psi}_{n}^{0}\right)\right) \\
& \quad-\sum_{l \in \mathcal{A}_{n}} n \lambda_{n}\left(\sqrt{\sum_{k=1}^{K} \tilde{w}_{n, k l}\left|\beta_{n, k l}^{0}+\alpha_{n} u_{k}\right|}-\sqrt{\sum_{k=1}^{K} \tilde{w}_{n, k l}\left|\beta_{n, k l}^{0}\right|}\right) \\
& \triangleq T_{n, 1}(\boldsymbol{u})-T_{n, 2}(\boldsymbol{u}) .
\end{aligned}
$$

To clarify, $l \in \mathcal{A}_{n}$ refers to all partly and completely uninformative covariates. From the proof of Theorem 1 we know that for $C$ large enough, $T_{n, 1}(\boldsymbol{u})$ is dominated by the term $-(1 / 2) n \alpha_{n}^{2} \boldsymbol{u}^{\prime} \mathcal{I}_{n}\left(\Psi_{n}^{0}\right) \boldsymbol{u}\left(1+o_{p}(1)\right)$. Also, since $\alpha_{n}=\sqrt{p_{n} / n} \rightarrow 0$, then for any $(k, l) \in \mathcal{A}_{n}$ and $n$ large enough we have $\left|\beta_{n, k l}^{0}+\alpha_{n} u_{k}\right| \geq\left|\beta_{n, k l}^{0}\right|-\left|\alpha_{n} u_{k}\right|>0$. Hence,

$$
\begin{aligned}
& \sqrt{\sum_{k=1}^{K} \tilde{w}_{n, k l}\left|\beta_{n, k l}^{0}+\alpha_{n} u_{k}\right|}-\sqrt{\sum_{k=1}^{K} \tilde{w}_{n, k l}\left|\beta_{n, k l}^{0}\right|} \\
& \quad \geq \sqrt{\sum_{k \in \mathcal{A}_{n}} \tilde{w}_{n, k l}\left|\beta_{n, k l}^{0}+\alpha_{n} u_{k}\right|}-\sqrt{\sum_{k \in \mathcal{A}_{n}} \tilde{w}_{n, k l}\left|\beta_{n, k l}^{0}\right|} \\
& \quad \geq \sqrt{\sum_{k \in \mathcal{A}_{n}} \tilde{w}_{n, k l}\left(\left|\beta_{n, k l}^{0}\right|-\left|\alpha_{n} u_{k}\right|\right)}-\sqrt{\sum_{k \in \mathcal{A}_{n}} \tilde{w}_{n, k l}\left|\beta_{n, k l}^{0}\right|} \\
& \geq \theta_{n}^{0}\left(\sqrt{1-\delta_{n}^{0}+O\left(\left(\delta_{n}^{0}\right)^{2}\right)}-1\right)
\end{aligned}
$$

where $\theta_{n}^{0}=\sqrt{\sum_{k \in \mathcal{A}_{n}} \tilde{w}_{n, k l}\left|\beta_{n, k l}^{0}\right|}$ and $\delta_{n}^{0}=\sum_{k \in \mathcal{A}_{n}} \tilde{w}_{n, k l}\left(\left|\alpha_{n} u_{k}\right|-\alpha_{n} u_{k}\left|\beta_{n, k l}^{0}\right|\right) /\left(\theta_{n}^{0}\right)^{2} \rightarrow$ 0 . From here, we adopt the same approach to Theorem 1 and show that the absolute 
80

$$
\left.\frac{\partial \ell_{n}\left(\mathbf{\Psi}_{n}\right)}{\partial \beta_{n, k l}}\right|_{\hat{\mathbf{\Psi}}_{n}}-0.5 n \lambda_{n} \tilde{w}_{n, k l} \operatorname{sgn}\left(\hat{\beta}_{n, k l}\right)\left(\sum_{k^{\prime}=1}^{K} \tilde{w}_{n, k^{\prime} l}\left|\hat{\beta}_{n, k^{\prime} l}\right|\right)^{-1 / 2}=0
$$

value of the $l^{\text {th }}$ summand in $-T_{n, 2}(\boldsymbol{u})$ is bounded by

$$
\begin{aligned}
\left|\left[-T_{n, 2}(\boldsymbol{u})\right]_{l}\right| & \leq\left|\frac{0.5 n \lambda_{n} \alpha_{n}\|\boldsymbol{u}\| \sum_{k \in \mathcal{A}_{n}} \tilde{w}_{n, k l} \sqrt{\tau_{2}}}{\sqrt{\sum_{k \in \mathcal{A}_{n}} \tilde{w}_{n, k l} \sqrt{\tau_{1}}}}\right|\left(1+o_{p}(1)\right) \\
& \leq\left|\frac{0.5 n \lambda_{n} \alpha_{n}\|\boldsymbol{u}\| \sqrt{a_{n} \tau_{2}}}{\sqrt[4]{\tau_{1}}}\right|\left(1+o_{p}(1)\right),
\end{aligned}
$$

where $\nu_{1}=\min \left\{\pi_{n, k}^{0} ; k \in \mathcal{A}_{n}\right\} \in(0,1)$. Under condition (A), we have $n \lambda_{n} \alpha_{n} \sqrt{a_{n}}=$ $o_{p}\left(n \alpha_{n}^{2}\right)$. Estimation consistency follows from this, since $D_{n}(\boldsymbol{u})$ will be less than 0 , with probability tending to 1 .

Next, we prove coefficient selection consistency. Suppose that for some $(k, l) \in \mathcal{B}_{n} \cup$ $\mathcal{C}_{n}$, we have $\hat{\beta}_{n, k l} \neq 0$. This implies

From the first part of the theorem, we know the first term on the left hand side of $(8)$ is $O_{p}\left(\sqrt{n p_{n}}\right)$. Using this order result, we can use (8) to further show that $n \lambda_{n}\left(\sum_{k^{\prime}=1}^{K} \tilde{w}_{n, k^{\prime} l}\left|\hat{\beta}_{n, k^{\prime} l}\right|\right)^{1 / 2}=\sum_{k^{\prime}=1}^{K} \hat{\beta}_{n, k^{\prime} l} O_{p}\left(\sqrt{n p_{n}}\right)$. Therefore, the second term on the left hand side of (8) can be written as

$$
0.5 n \lambda_{n} \tilde{w}_{n, k l} \operatorname{sgn}\left(\hat{\beta}_{n, k l}\right)\left(\sum_{k^{\prime}=1}^{K} \tilde{w}_{n, k^{\prime} l}\left|\hat{\beta}_{n, k^{\prime} l}\right|\right)^{-1 / 2}=\frac{0.5\left(n \lambda_{n}\right)^{2} \tilde{w}_{n, k l} \operatorname{sgn}\left(\hat{\beta}_{n, k l}\right)}{\sum_{k^{\prime}=1}^{K} \hat{\beta}_{n, k^{\prime} l} O_{p}\left(\sqrt{n p_{n}}\right)} .
$$

For $\left(k^{\prime}, l\right) \in \mathcal{A}_{n}$, i.e. a non-zero coefficient in a partly uninformative covariate, $\hat{\beta}_{n, k^{\prime} l}=O_{p}(1)$. Otherwise, if $\left(k^{\prime}, l\right) \in \mathcal{B}_{n} \cup \mathcal{C}_{n}$, i.e. a zero coefficient in a partly or completely uninformative covariate, then $\hat{\beta}_{n, k^{\prime} l}=O_{p}\left(\sqrt{p_{n} / n}\right)$. Since $p_{n}=o_{p}\left(\sqrt{n p_{n}}\right)$ by condition $(\mathrm{C})$, then we have

$$
\left|\frac{0.5\left(n \lambda_{n}\right)^{2} \tilde{w}_{n, k l} \operatorname{sgn}\left(\hat{\beta}_{n, k l}\right)}{\sum_{k^{\prime}=1}^{K} \hat{\beta}_{n, k^{\prime} l} O_{p}\left(\sqrt{n p_{n}}\right)}\right| \geq\left|\frac{0.5\left(n \lambda_{n}\right)^{2} b_{n} \operatorname{sgn}\left(\hat{\beta}_{n, k l}\right)}{O_{p}\left(\sqrt{n p_{n}}\right)}\right|=O_{p}\left(n^{3 / 2} \lambda_{n}^{2} b_{n} / \sqrt{p_{n}}\right) .
$$

Hence by condition (B), the second term in (8) dominates the first term as $n \rightarrow 0$. Since $\sqrt{n p_{n}} \rightarrow \infty$, it follows that the equality in (8) cannot hold with probability tending to 1 , and thus $\hat{\beta}_{n, k l}=0$ for all $(k, l) \in \mathcal{B}_{n} \cup \mathcal{C}_{n}$.

The proof of asymptotic normality is very similar to the corresponding proof in Theorem 1, and so we have omitted it here. 


\section{APPENDIX B: ESTIMATION PROCEDURE}

$$
Q_{n}^{\text {pen }}(\boldsymbol{\Psi})=\sum_{i=1}^{n} \sum_{k=1}^{K} \hat{\tau}_{i k}^{(t)}\left(\log \pi_{k}+\log f\left(y_{i} ; \boldsymbol{x}_{i}, \mu_{i k}, \phi_{k}\right)\right)-n \lambda_{n} \sum_{l=1}^{p_{n}} \mathcal{P}\left(\boldsymbol{\beta}_{l}\right),
$$

109 110

We compute our penalized estimates using a hybrid Expectation Maximization (EM) algorithm, which combines the standard EM procedure (Dempster et al., 1977) with a local quadratic approximation (LQA, Fan and Li, 2001). Let $\boldsymbol{z}_{i}=\left(z_{i 1}, \ldots, z_{i K}\right)^{\prime}$ denote the unobserved group-label for observation $i$, where $z_{i k}=1$ if observation $i$ belongs to component $k$ and 0 otherwise. We define the penalized complete loglikelihood function $\ell_{n, C}^{\text {pen }}(\boldsymbol{\Psi})=\ell_{n, C}(\boldsymbol{\Psi})-n \lambda_{n} \sum_{l=1}^{p} \mathcal{P}\left(\boldsymbol{\beta}_{l}\right)$, where $\ell_{n, C}(\boldsymbol{\Psi})=\sum_{i=1}^{n} \sum_{k=1}^{K} z_{i k}\left(\log \pi_{k}+\log f\left(y_{i} ; \boldsymbol{x}_{i}, \mu_{i k}, \phi_{k}\right)\right)$. The EM algorithm iterates between the following two steps until convergence: Give the estimates $\hat{\Psi}^{(t)}$ at the $t^{\text {th }}$ iteration,

E-Step: Calculate the Q-function $Q_{n}^{\text {pen }}(\boldsymbol{\Psi})=E_{\boldsymbol{Z} \mid \boldsymbol{\Psi}}\left(\ell_{n, C}^{\text {pen }}(\boldsymbol{\Psi}) \mid \hat{\mathbf{\Psi}}^{(t)}\right)$,

where $\hat{\tau}_{i k}^{(t)}$ is the posterior probability of observation $i$ belong to component $k$, evaluated at the current estimates

$$
\hat{\tau}_{i k}^{(t)}=\frac{\hat{\pi}_{k}^{(t)} f\left(y_{i} ; \boldsymbol{x}_{i}, \hat{\mu}_{i k}^{(t)}, \hat{\phi}_{k}^{(t)}\right)}{\sum_{k^{\prime}=1}^{K} \hat{\pi}_{k^{\prime}}^{(t)} f\left(y_{i} ; \boldsymbol{x}_{i}, \hat{\mu}_{i k^{\prime}}^{(t)}, \hat{\phi}_{k^{\prime}}^{(t)}\right)}
$$

M-step: Maximize the Q-function to obtain $\hat{\Psi}^{(t+1)}=\arg \max _{\Psi} Q_{n}^{\text {pen }}(\mathbf{\Psi})$.

Maximizing the Q-function with respect to $\boldsymbol{\phi}$ and $\boldsymbol{\pi}$ are straightforward, since $\mathcal{P}\left(\boldsymbol{\beta}_{l}\right)$ does not depend on the these sets of parameters. To accomplish the maximization with respect to $\boldsymbol{\beta}$, we apply the LQA: At iteration $t$, set $\hat{\beta}_{k l}^{(t+1)}=0$ if $\hat{\beta}_{k l}^{(t)}$ is close to 0 (e.g., if $\left|\hat{\beta}_{k l}^{(t)}\right|<0.001$ ), otherwise we approximate the penalty as

$$
\mathcal{P}\left(\boldsymbol{\beta}_{l}\right) \approx \mathcal{P}\left(\hat{\boldsymbol{\beta}}_{l}^{(t)}\right)+\frac{1}{2}\left(\boldsymbol{\beta}_{l}^{\prime} \boldsymbol{S}_{l}^{(t)} \boldsymbol{\beta}_{l}-\hat{\boldsymbol{\beta}}_{l}^{(t)^{\prime}} \boldsymbol{S}_{l}^{(t)} \hat{\boldsymbol{\beta}}_{l}^{(t)}\right) \propto \frac{1}{2} \sum_{k=1}^{K}\left[\boldsymbol{S}_{l}^{(t)}\right]_{k k}\left(\beta_{k l}^{2}-\hat{\beta}_{k l}^{(t) 2}\right)
$$

${ }_{116}$ where $\boldsymbol{S}_{l}^{(t)}$ is a diagonal matrix with $\left[\boldsymbol{S}_{l}^{(t)}\right]_{k k}=\left(1 /\left|\beta_{k l}^{(t)}\right|\right) \times \partial \mathcal{P}\left(\boldsymbol{\beta}_{l}^{(t)}\right) / \partial\left|\beta_{k l}\right|$. In the 117 case of the MIXGL2 and MIXGL1 penalties, these leads to the following forms,

$$
\text { For MIXGL2: } \quad\left[\boldsymbol{S}_{l}^{(t)}\right]_{k k}=\frac{\tilde{w}_{l}}{\sqrt{\sum_{k=1}^{K} \hat{\beta}_{k l}^{2(t)}}}
$$




$$
\text { For MIXGL1: } \quad\left[\boldsymbol{S}_{l}^{(t)}\right]_{k k}=\frac{\tilde{w}_{k l}}{2\left|\hat{\beta}_{k l}^{(t)}\right| \sqrt{\sum_{k=1}^{K} \tilde{w}_{k l}\left|\hat{\beta}_{k l}^{(t)}\right|}}
$$

for Maximization in the M-step can now be performed on a per-component basis as follows:

M-Step [LQA]: Maximize the approximated Q-function

$$
Q_{n}^{\text {pen }}(\boldsymbol{\Psi})=\sum_{i=1}^{n} \sum_{k=1}^{K} \hat{\tau}_{i k}^{(t)}\left(\log \pi_{k}+\log f\left(y_{i} ; \boldsymbol{x}_{i}, \mu_{i k}, \phi_{k}\right)\right)-\frac{1}{2} n \lambda_{n} \sum_{k=1}^{K} \sum_{l=1}^{p_{n}}\left[\boldsymbol{S}_{l}^{(t)}\right]_{k k} \beta_{k l}^{2} .
$$

By iterating between the E-step and modified M-step, the MIXGL2 and MIXGL1 estimates are straightforwardly computed. In our simulations and application, we used the unpenalized maximum likelihood estimates from the full model as starting values in our algorithm. Besides, the full model needs to be fitted in order to obtain the weights in the MIXGL2 and MIXGL1 penalties.

A proof that the above hybrid EM-LQA algorithm possesses the ascent property, that is, each M-step leads to an increase in the penalized log-likelihood, is provided at the end of Appendix B.

Estimation for SAMs. For SAMs defined in equation (2) of the main text, the penalized complete log-likelihood function is given by,

$$
\begin{aligned}
\ell_{n, C}^{\text {pen }}(\boldsymbol{\Psi}) & =\ell_{n, C}(\boldsymbol{\Psi})-\lambda_{n} \sum_{l=1}^{p} \mathcal{P}\left(\boldsymbol{\beta}_{l}\right) \\
& =\sum_{j=1}^{s} \sum_{k=1}^{K} z_{j k}\left(\log \pi_{k}+\sum_{i=1}^{n} \log \left(\mu_{i j k}^{y_{i j}}\left(1-\mu_{i j k}\right)^{1-y_{i j}}\right)\right)-\lambda_{n} \sum_{l=1}^{p} \mathcal{P}\left(\boldsymbol{\beta}_{l}\right),
\end{aligned}
$$

where $z_{j k}=1$ is species $j$ belongs to archetype $k$. Note the parametrization of the tuning parameter now as $\lambda_{n}$ instead of $n \lambda_{n}$. The hybrid EM algorithm described above can be straightforwardly extended to the case of SAMs. Specifically, at iteration $t$ the E-step replaces $z_{i k}$ by the posterior probabilities,

$$
\hat{\tau}_{j k}^{(t)}=\frac{\hat{\pi}_{k}^{(t)} \prod_{i=1}^{n}\left(\hat{\mu}_{i j k}^{(t)}\right)^{y_{i j}}\left(1-\hat{\mu}_{i j k}^{(t)}\right)^{1-y_{i j}}}{\sum_{k^{\prime}=1}^{K} \hat{\pi}_{k^{\prime}}^{(t)} \prod_{i=1}^{n}\left(\hat{\mu}_{i j k^{\prime}}^{(t)}\right)^{y_{i j}}\left(1-\hat{\mu}_{i j k^{\prime}}^{(t)}\right)^{1-y_{i j}}} .
$$

To perform the M-step, we apply the LQA, leading to, M-Step [LQA]: Maximize the approximated Q-function

$Q_{n}^{\text {pen }}(\boldsymbol{\Psi})=\sum_{j=1}^{s} \sum_{k=1}^{K} \hat{\tau}_{j k}^{(t)}\left(\log \pi_{k}+\sum_{i=1}^{n} \log \left(\mu_{i j k}^{y_{i j}}\left(1-\mu_{i j k}\right)^{1-y_{i j}}\right)\right)-\frac{1}{2} \lambda_{n} \sum_{k=1}^{K} \sum_{l=1}^{p_{n}}\left[\boldsymbol{S}_{l}^{(t)}\right]_{k k} \beta_{k l}^{2}$. 
The maximization can then be performed on a conditional basis (leading to the Expectation Conditional Maximization or ECM algorithm, Meng and Rubin, 1993) as follows: conditional on the species-specific intercepts $\beta_{0 j} ; j=1, \ldots, s$, update the archetypal regression coefficients on a per-archetype basis. Then conditional on the updated regression coefficients, update the species-specific intercepts $\beta_{0 j} ; j=$ $1, \ldots, s$. Note the intercepts are not penalized, meaning their updating can be done as a $s$ lots of univariate updates (which themselves can be performed using an optimization routine such as Nelder-Mead for instance, Nelder and Mead, 1965).

Proof of Ascent Property for Hybrid EM-LQA Algorithm. For ease of presentation, we use a slightly modified notation compared to the proofs presented in Appendix A.

Let $\ell(\boldsymbol{\Psi})$ denote the marginal log-likelihood of the mixture model, $p_{\lambda}(|\boldsymbol{\Psi}|)$ denote the chosen penalty function (whether it be MIXGL1, MIXGL2, or some other penalty), and $\ell_{\text {pen }}(\boldsymbol{\Psi})=\ell(\boldsymbol{\Psi})-p_{\lambda}(|\boldsymbol{\Psi}|)$. Suppose that at iteration $t$ of the hybrid EM-LQA algorithm, we have estimates $\boldsymbol{\Psi}_{(t)}$. Furthermore, we can calculate the (unpenalized) Q-function,

$$
Q\left(\boldsymbol{\Psi} \mid \boldsymbol{\Psi}_{(t)}\right)=E_{\boldsymbol{Z} \mid \boldsymbol{\Psi}}\left(\ell(\boldsymbol{\Psi}) \mid \boldsymbol{\Psi}_{(t)}\right),
$$

as well as the LQA at $\boldsymbol{\Psi}_{(t)}$,

$$
\tilde{p}_{\lambda}\left(\boldsymbol{\Psi} \mid \boldsymbol{\Psi}_{(t)}\right)=p_{\lambda}\left(\left|\Psi_{(t)}\right|\right)+\frac{1}{2} \frac{p_{\lambda}^{\prime}\left(\left|\boldsymbol{\Psi}_{(t)}\right|\right)}{\left|\boldsymbol{\Psi}_{(t)}\right|}\left(\boldsymbol{\Psi}^{2}-\boldsymbol{\Psi}_{(t)}^{2}\right),
$$

where for ease of exposition the use of vector notation has been suppressed. Furthermore, a crucial property we utilize below is the fact that the LQA majorizes the penalty at $\boldsymbol{\Psi}_{(t)}$, that is $\tilde{p}_{\lambda}\left(\boldsymbol{\Psi} \mid \boldsymbol{\Psi}_{(t)}\right) \geq p_{\lambda}(|\boldsymbol{\Psi}|)$ for all $\boldsymbol{\Psi}$ with equality when $\boldsymbol{\Psi}=\boldsymbol{\Psi}_{(t)}$ (Hunter and $\mathrm{Li}, 2005$ ).

Consider the following inequality

$$
\begin{aligned}
\ell_{p}(\boldsymbol{\Psi})-\ell_{p}\left(\boldsymbol{\Psi}_{(t)}\right) & =\ell(\boldsymbol{\Psi})-\ell\left(\boldsymbol{\Psi}_{(t)}\right)-\left(p_{\lambda}(|\boldsymbol{\Psi}|)-p_{\lambda}\left(\left|\mathbf{\Psi}_{(t)}\right|\right)\right) \\
& \geq Q\left(\boldsymbol{\Psi} \mid \boldsymbol{\Psi}_{(t)}\right)-Q\left(\boldsymbol{\Psi}_{(t)} \mid \boldsymbol{\Psi}_{(t)}\right)-\left(p_{\lambda}(|\boldsymbol{\Psi}|)-p_{\lambda}\left(\left|\boldsymbol{\Psi}_{(t)}\right|\right)\right) \\
& \geq Q\left(\boldsymbol{\Psi} \mid \mathbf{\Psi}_{(t)}\right)-Q\left(\boldsymbol{\Psi}_{(t)} \mid \boldsymbol{\Psi}_{(t)}\right)-\left(\tilde{p}_{\lambda}\left(\boldsymbol{\Psi} \mid \mathbf{\Psi}_{(t)}\right)-p_{\lambda}\left(\left|\mathbf{\Psi}_{(t)}\right|\right)\right)
\end{aligned}
$$

where the second line follows from the ascent property of the standard EM algorithm (see Wikipedia, 2015, under Proof of correctness), and the third line follows from the majorization property mentioned above. Following some straightforward algebra, we have

$\ell_{p}(\boldsymbol{\Psi})-\ell_{p}\left(\boldsymbol{\Psi}_{(t)}\right) \geq\left(Q\left(\boldsymbol{\Psi} \mid \boldsymbol{\Psi}_{(t)}\right)-\frac{1}{2} \frac{p_{\lambda}^{\prime}\left(\left|\boldsymbol{\Psi}_{(t)}\right|\right)}{\left|\boldsymbol{\Psi}_{(t)}\right|} \boldsymbol{\Psi}^{2}\right)-\left(Q\left(\boldsymbol{\Psi}_{(t)} \mid \boldsymbol{\Psi}_{(t)}\right)-\frac{1}{2} \frac{p_{\lambda}^{\prime}\left(\left|\boldsymbol{\Psi}_{(t)}\right|\right)}{\left|\boldsymbol{\Psi}_{(t)}\right|} \boldsymbol{\Psi}_{(t)}^{2}\right)$. 
${ }_{163}$ The above inequality implies that if one can find the penalized estimate $\boldsymbol{\Psi}^{(t+1)}$ which 164 maximizes $Q\left(\boldsymbol{\Psi} \mid \boldsymbol{\Psi}_{(t)}\right)-0.5\left(p_{\lambda}^{\prime}\left(\left|\boldsymbol{\Psi}_{(t)}\right|\right) /\left|\boldsymbol{\Psi}_{(t)}\right|\right) \boldsymbol{\Psi}^{2}$, i.e. maximizing the approximated 165 penalized Q-function, denoted as $Q_{n}^{\text {pen }}(\boldsymbol{\Psi})$ earlier, then $\ell_{p}\left(\Psi^{(t+1)}\right) \geq \ell_{p}\left(\Psi_{(t)}\right)$ and 166 the ascent property of the penalized log-likelihood is therefore guaranteed.

\section{APPENDIX C: ADDITIONAL RESULTS FOR SECTION 4}

167 We first present maps showing the predicted probabilities of presence, on the linear predictor scale, for each archetype in both penalized SAMs. As stated in the main text, we mapped the linear predictors $\hat{\eta}_{i k}$ directly rather convert them to probabilities (via the inverse-logit or logistic function), as this typically makes it easier to recognize any differences between the archetypes. Following these two sets of maps, we provide tables of the estimated coefficients for the MIXGL2 and MIXGL1 SAMs. 
Probability of Presence - Archetype 1 (MIXGL2)

173

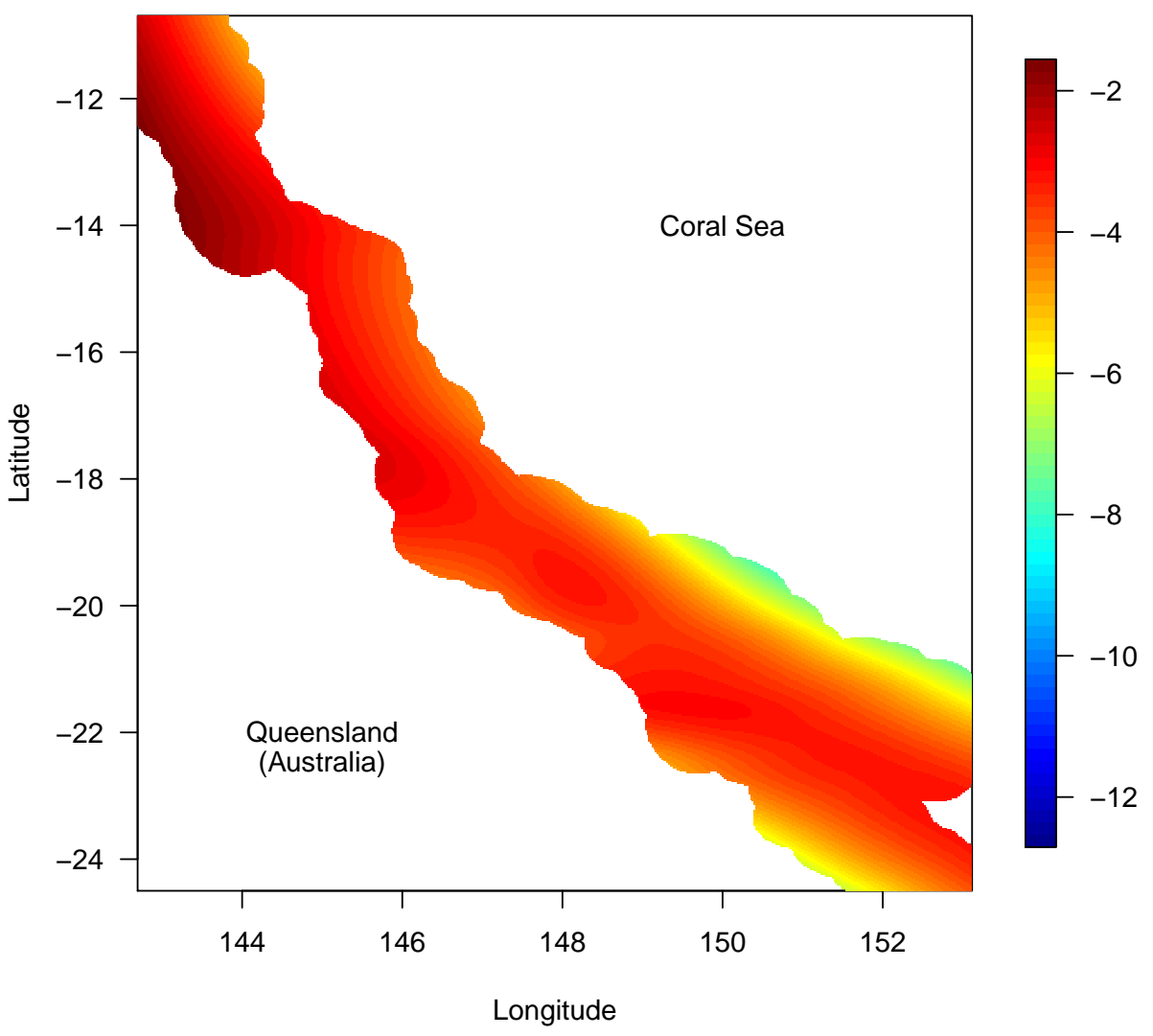

Probability of Presence - Archetype 2 (MIXGL2)

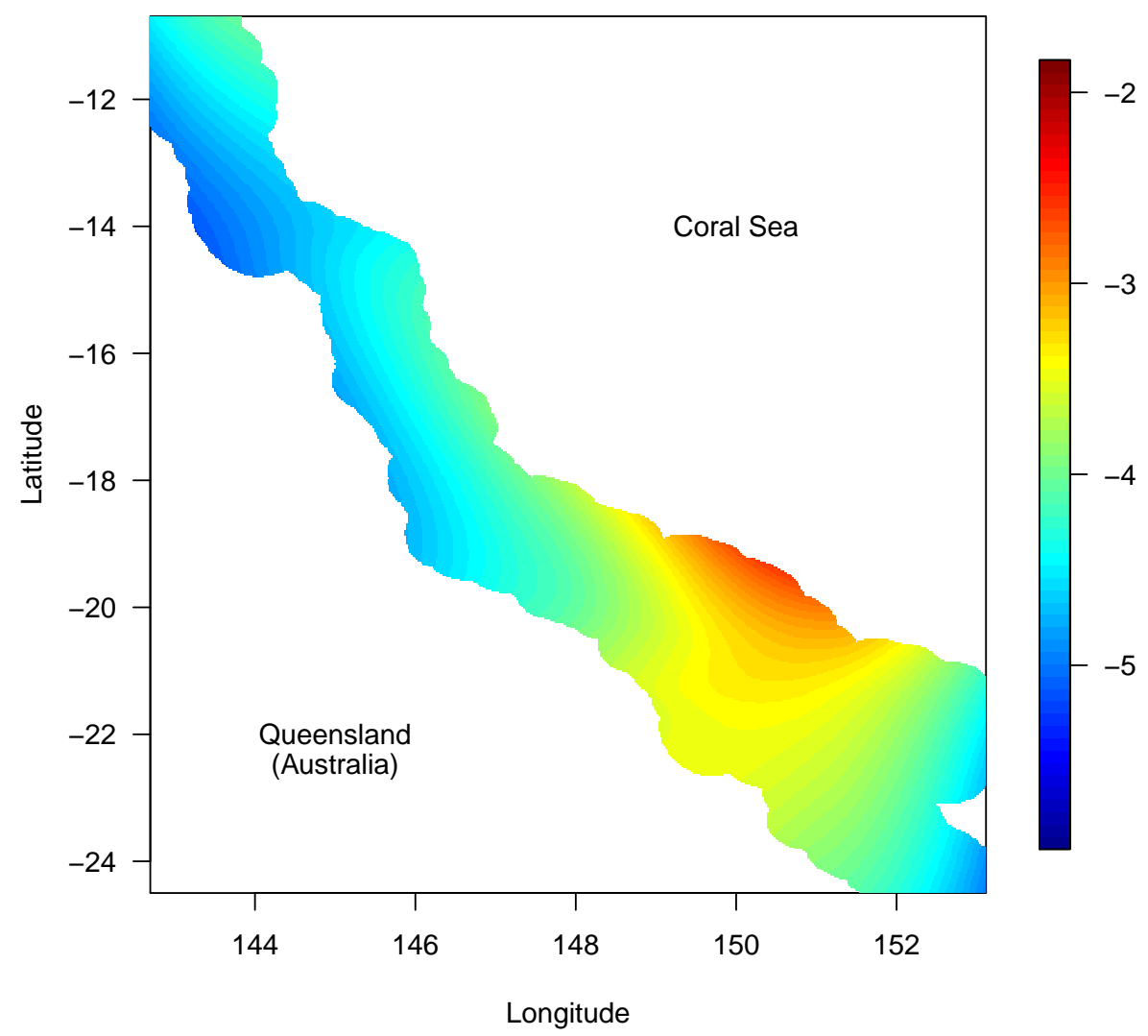


Probability of Presence - Archetype 3 (MIXGL2)

174

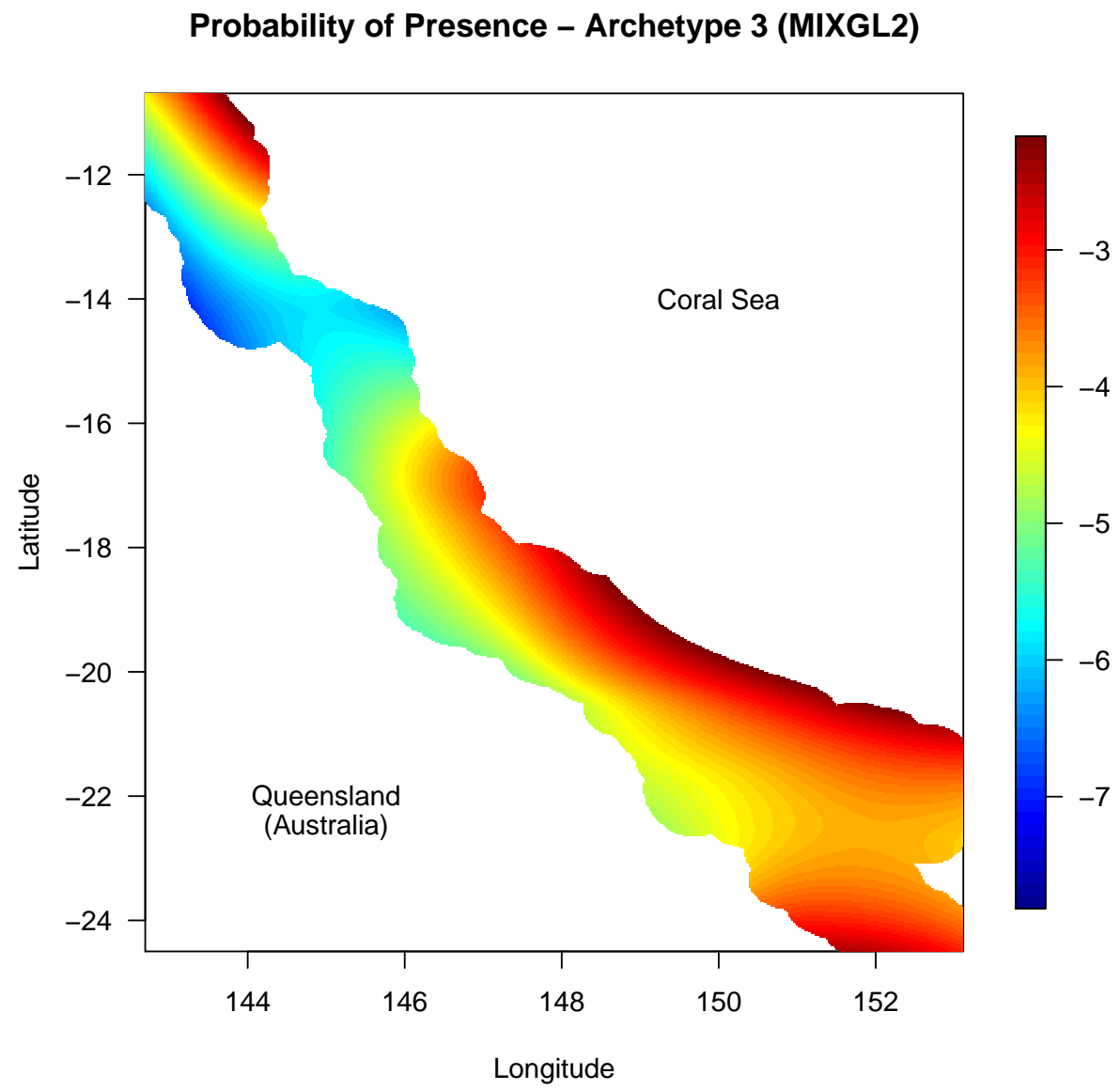

Probability of Presence - Archetype 4 (MIXGL2)

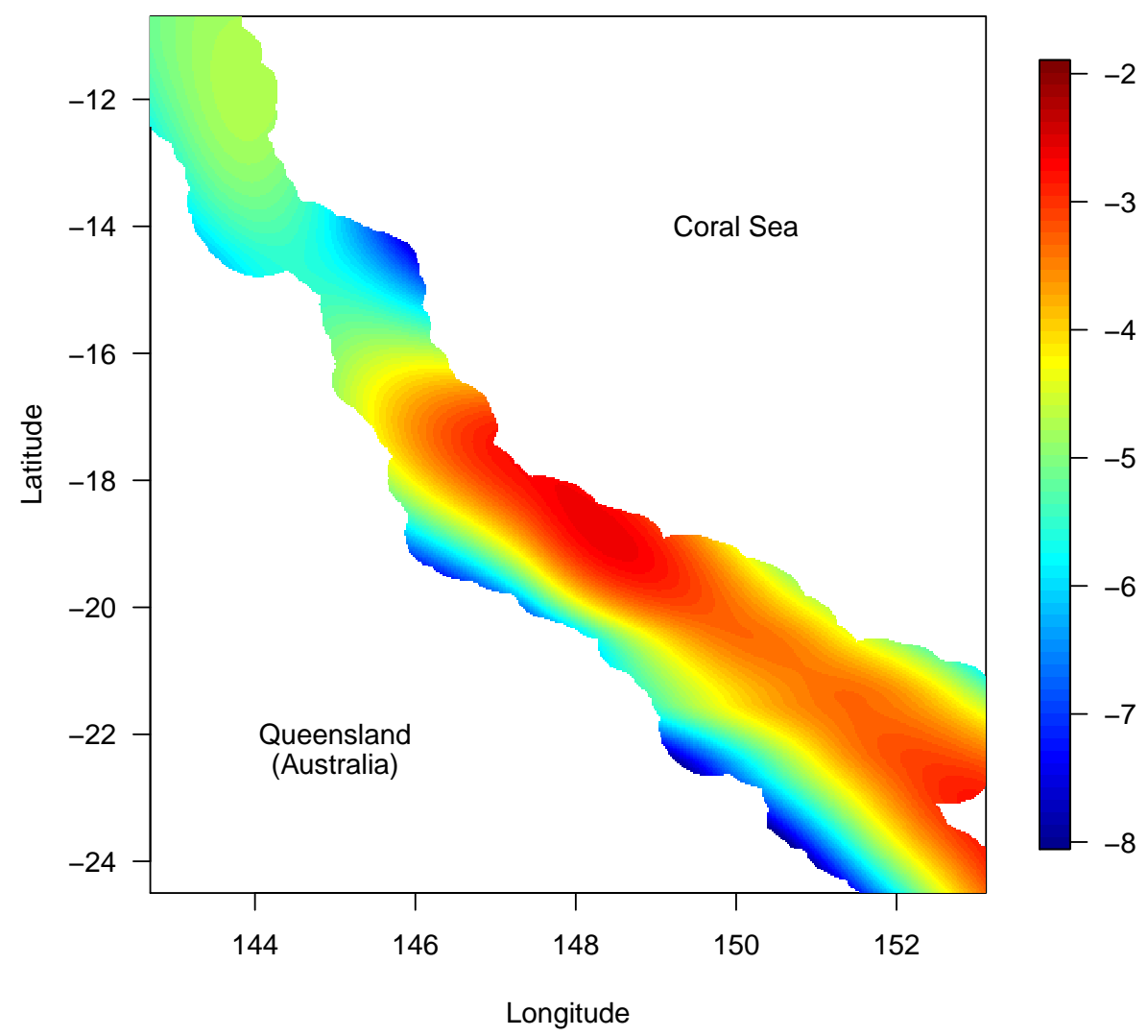


Probability of Presence - Archetype 5 (MIXGL2)

175

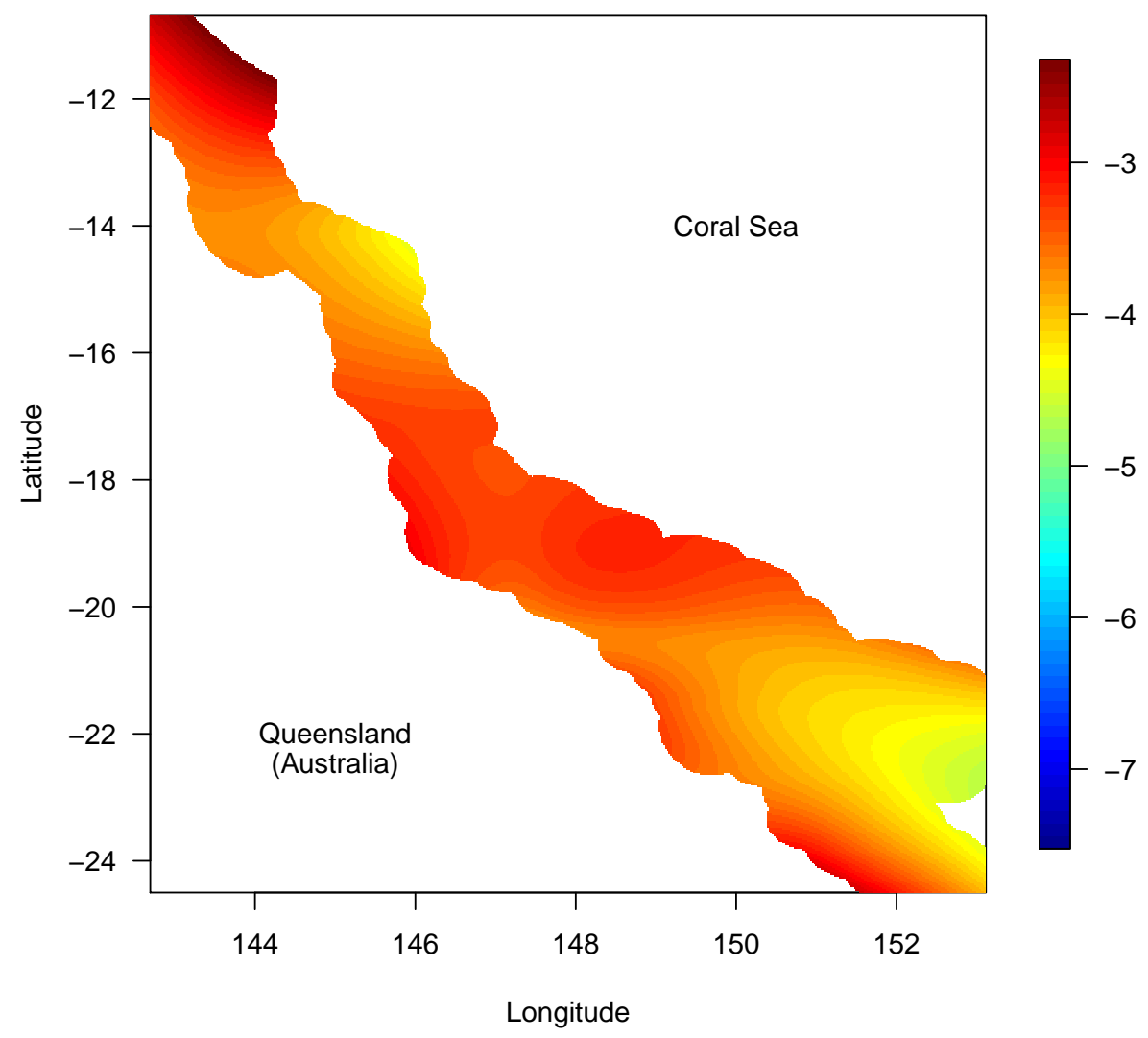

Probability of Presence - Archetype 6 (MIXGL2)

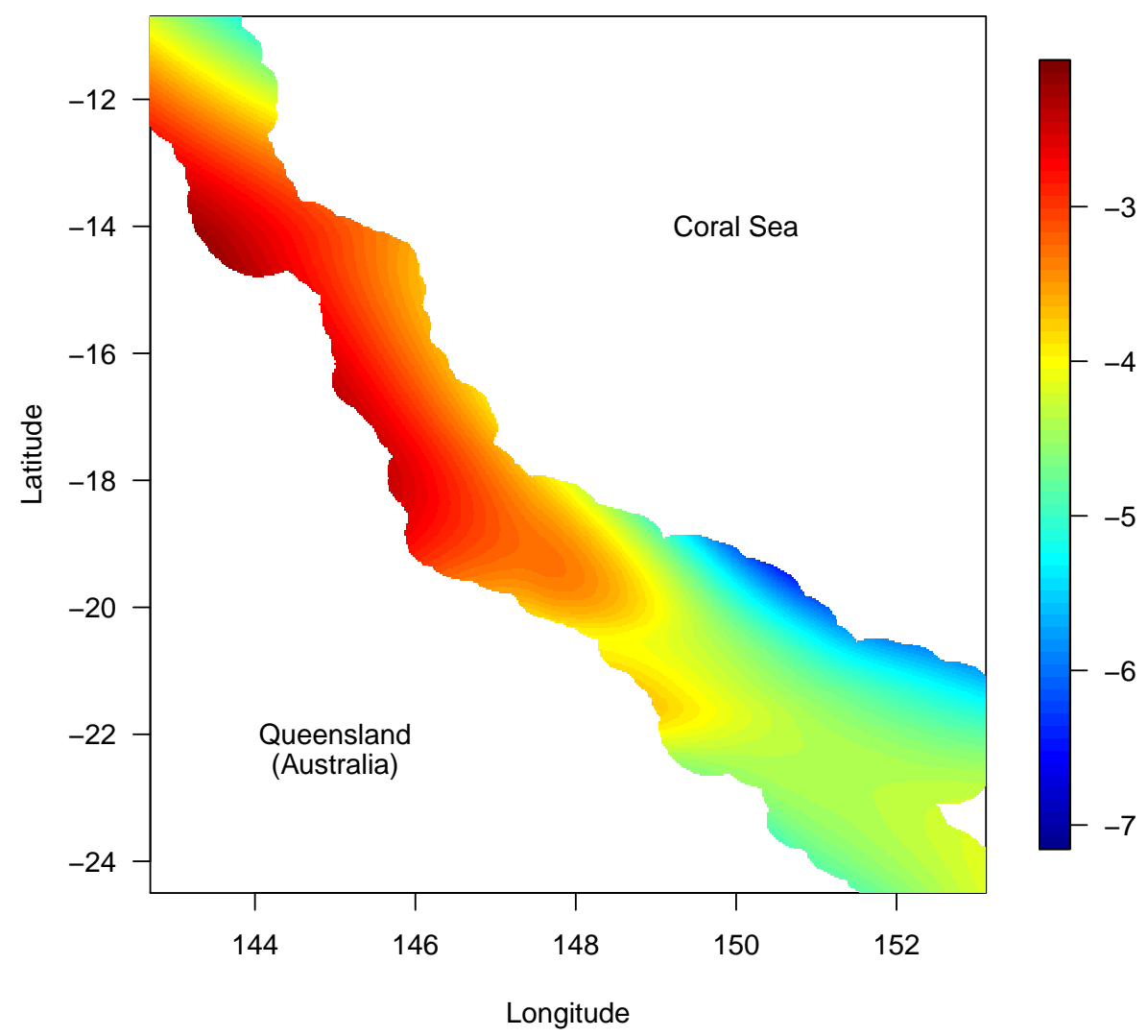


Probability of Presence - Archetype 7 (MIXGL2)

176

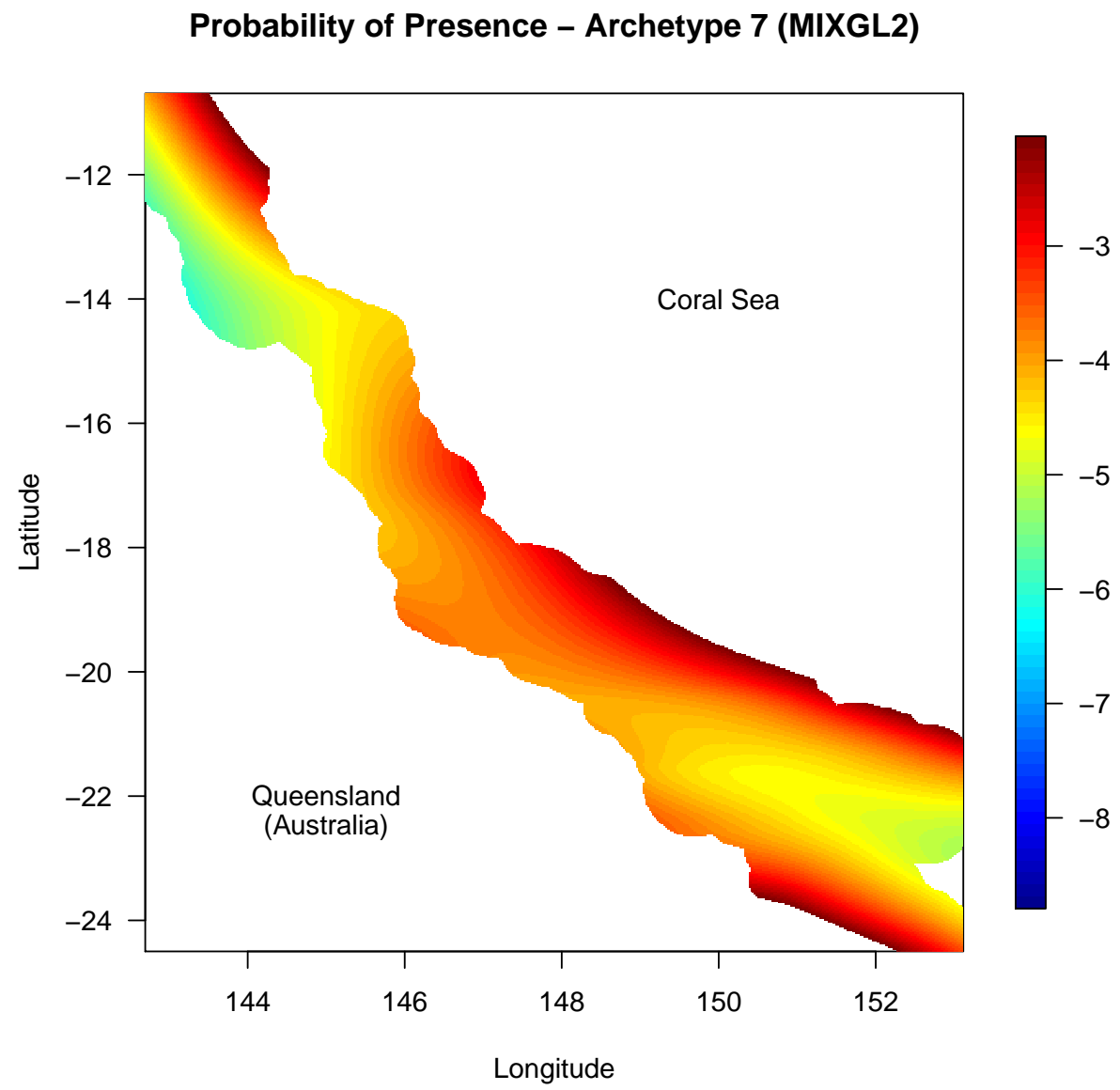

Probability of Presence - Archetype 8 (MIXGL2)

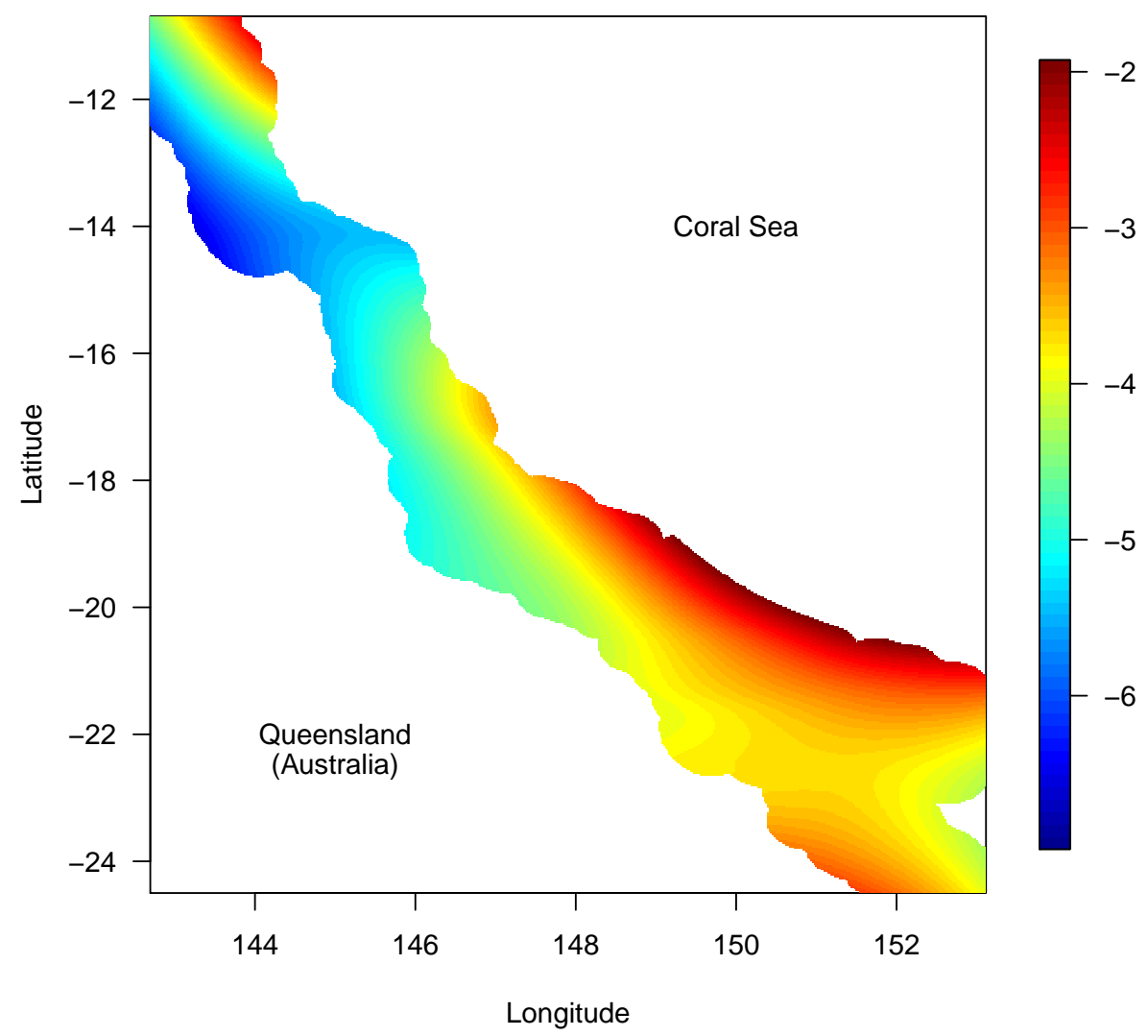


Probability of Presence - Archetype 9 (MIXGL2)

177

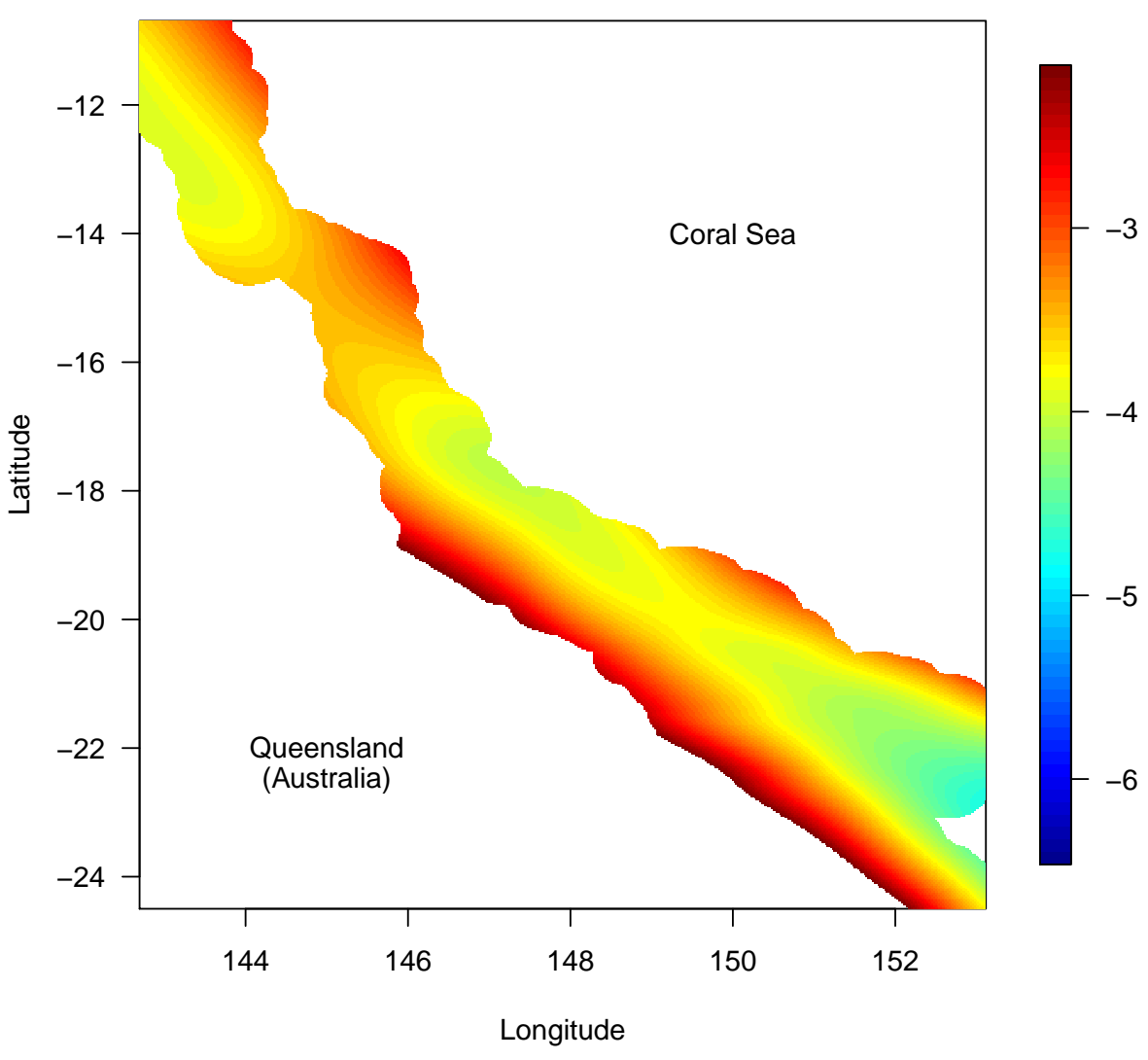


Probability of Presence - Archetype 1 (MIXGL1)

178

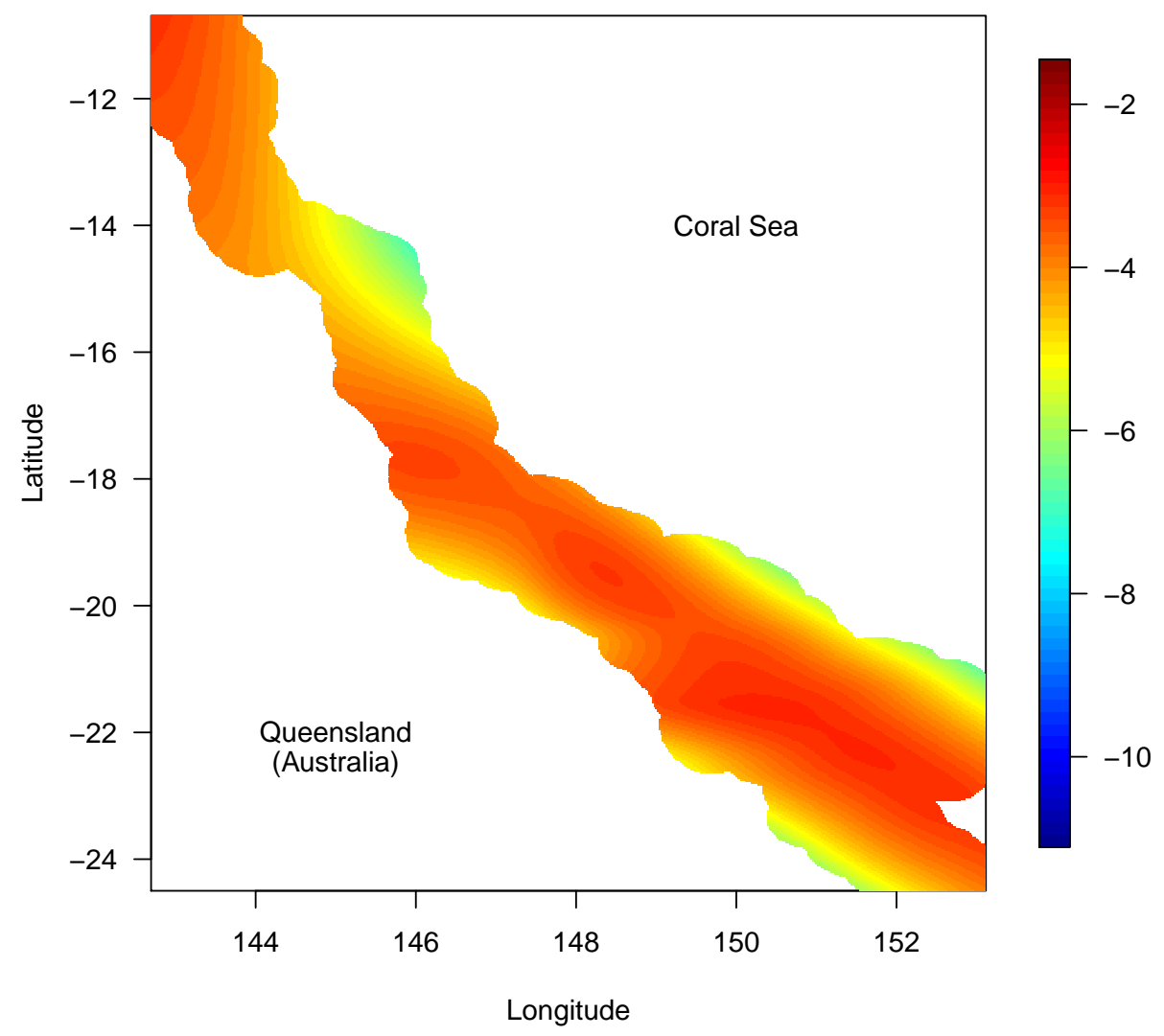

Probability of Presence - Archetype 2 (MIXGL1)

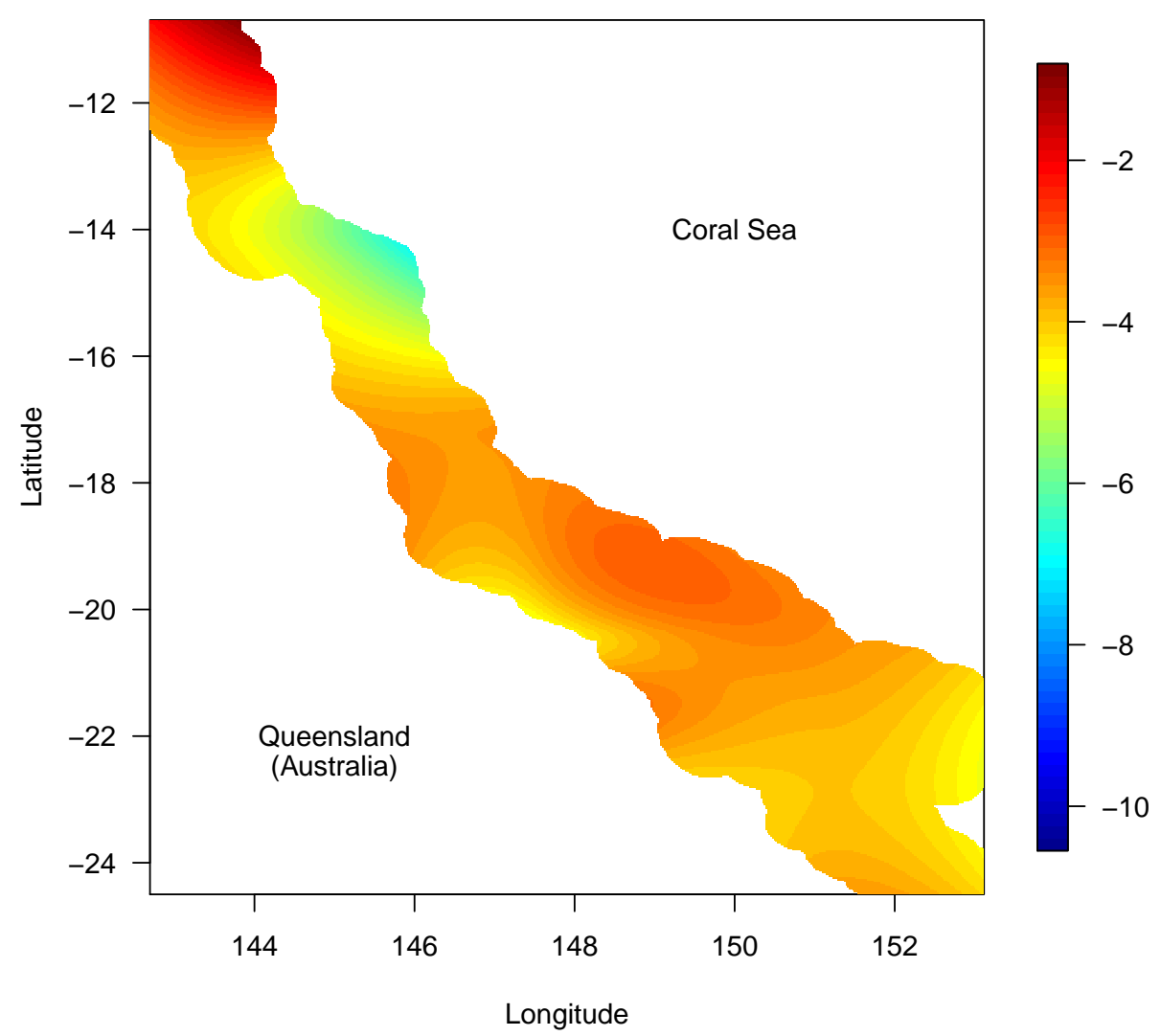


Probability of Presence - Archetype 3 (MIXGL1)

179

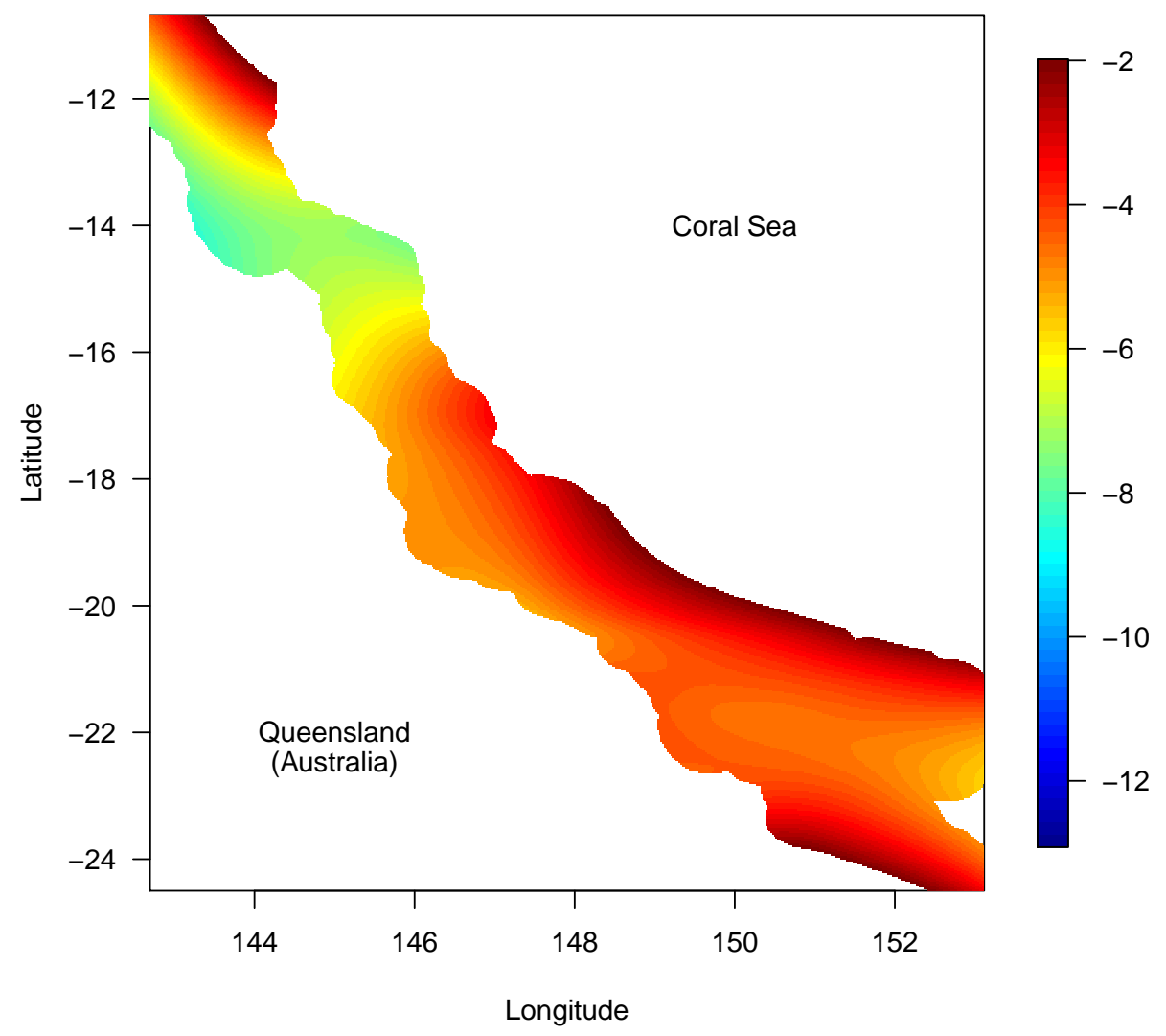

Probability of Presence - Archetype 4 (MIXGL1)

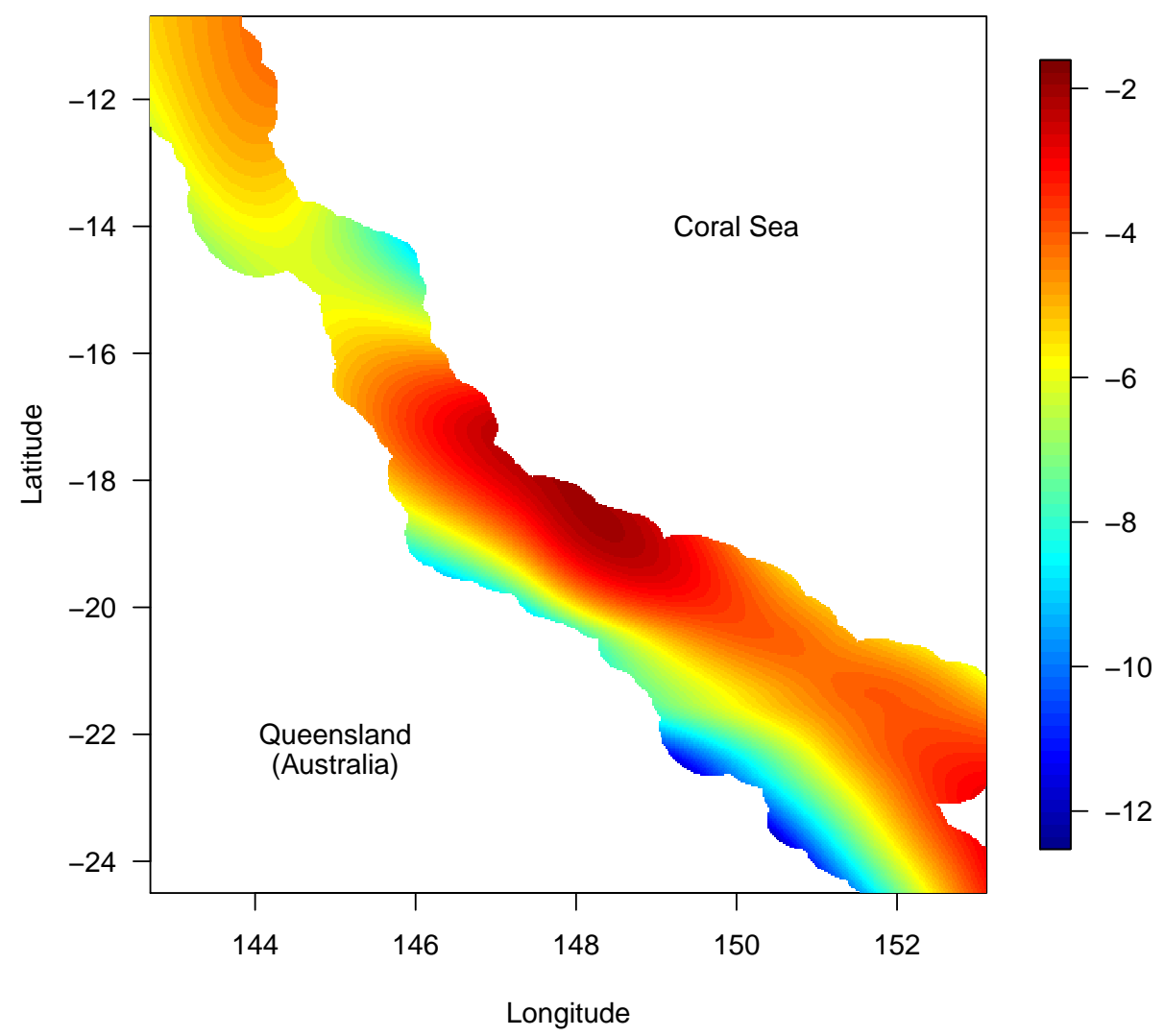


Probability of Presence - Archetype 5 (MIXGL1)

180

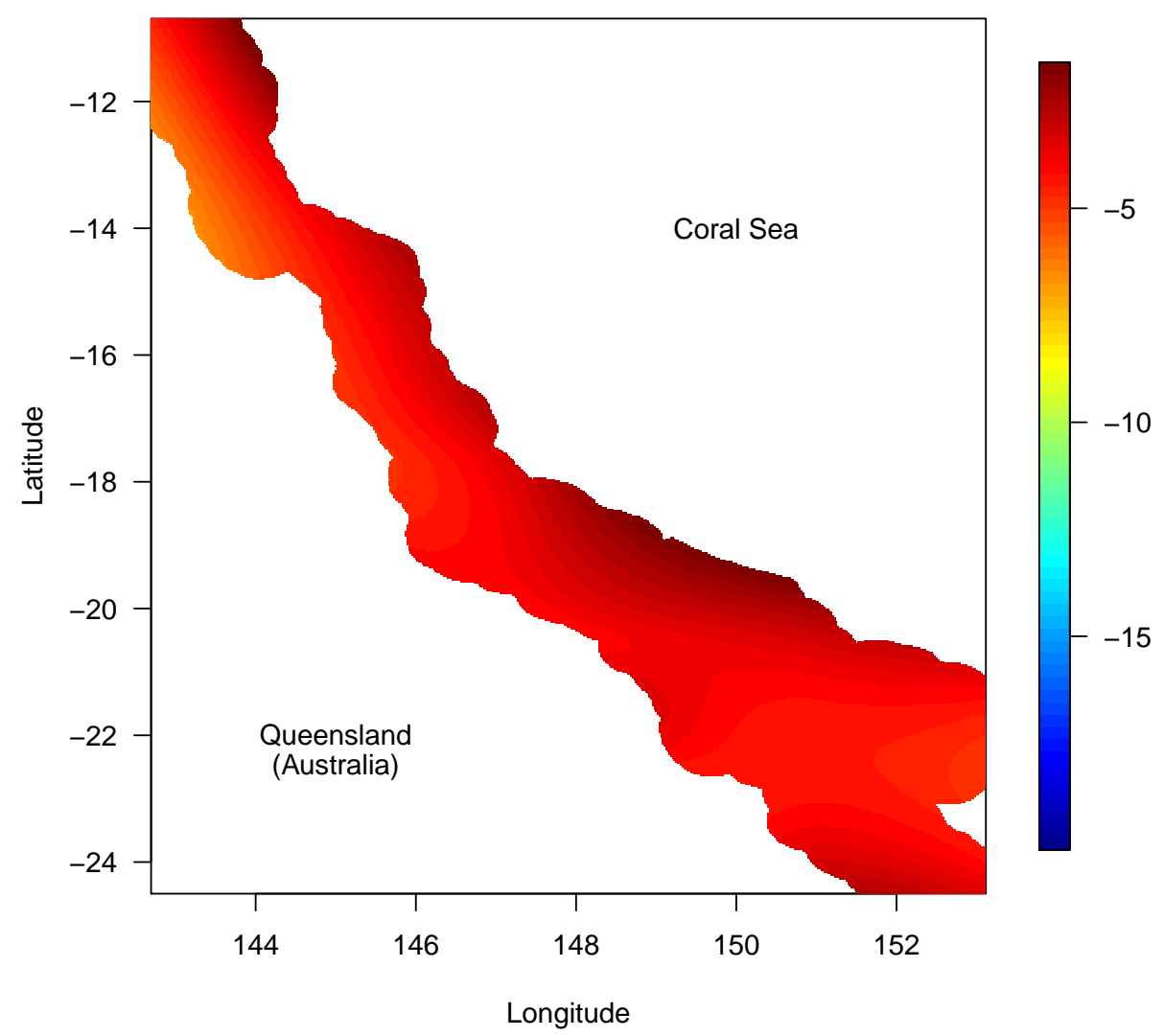

Probability of Presence - Archetype 6 (MIXGL1)

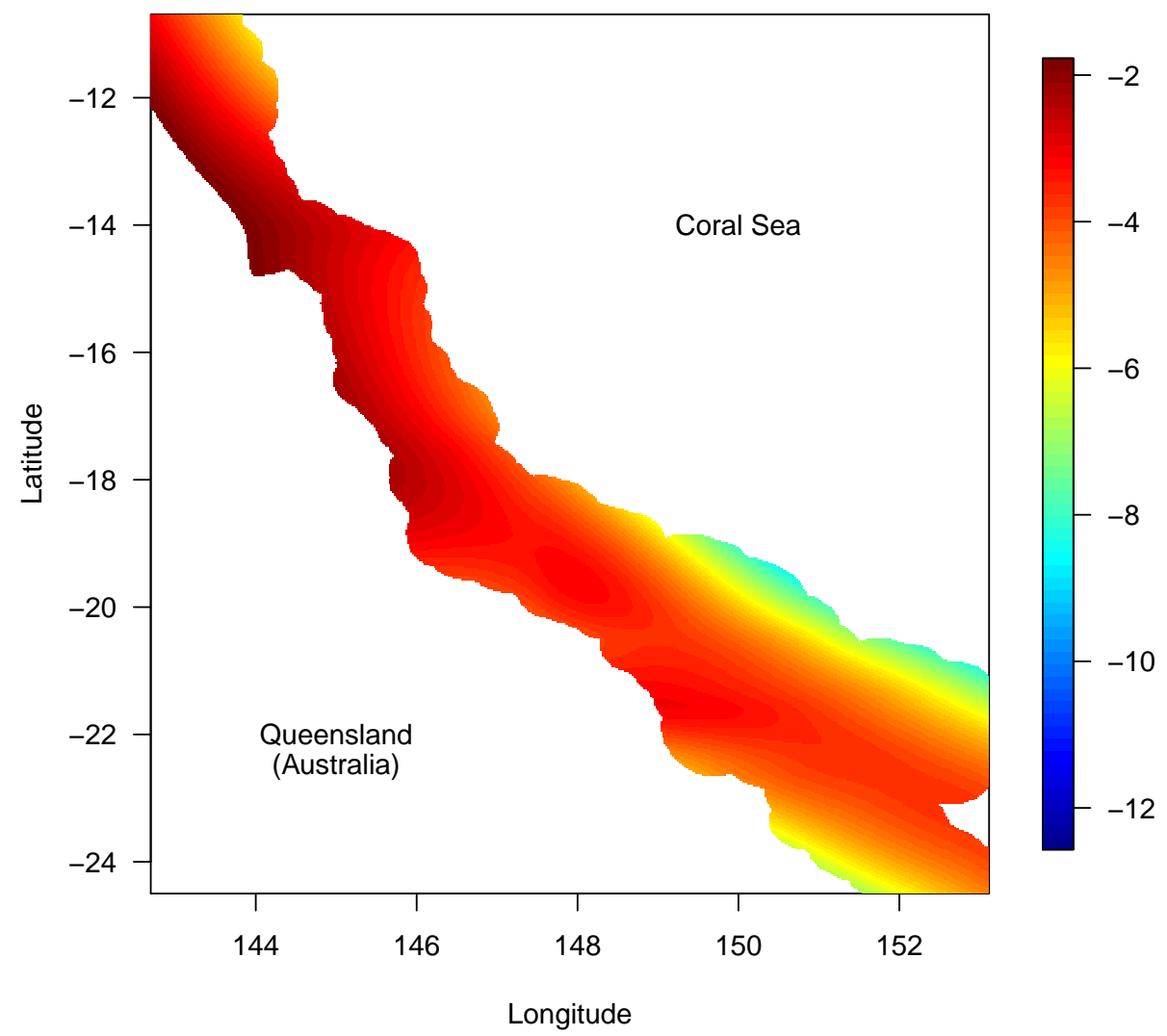


Probability of Presence - Archetype 7 (MIXGL1)

181

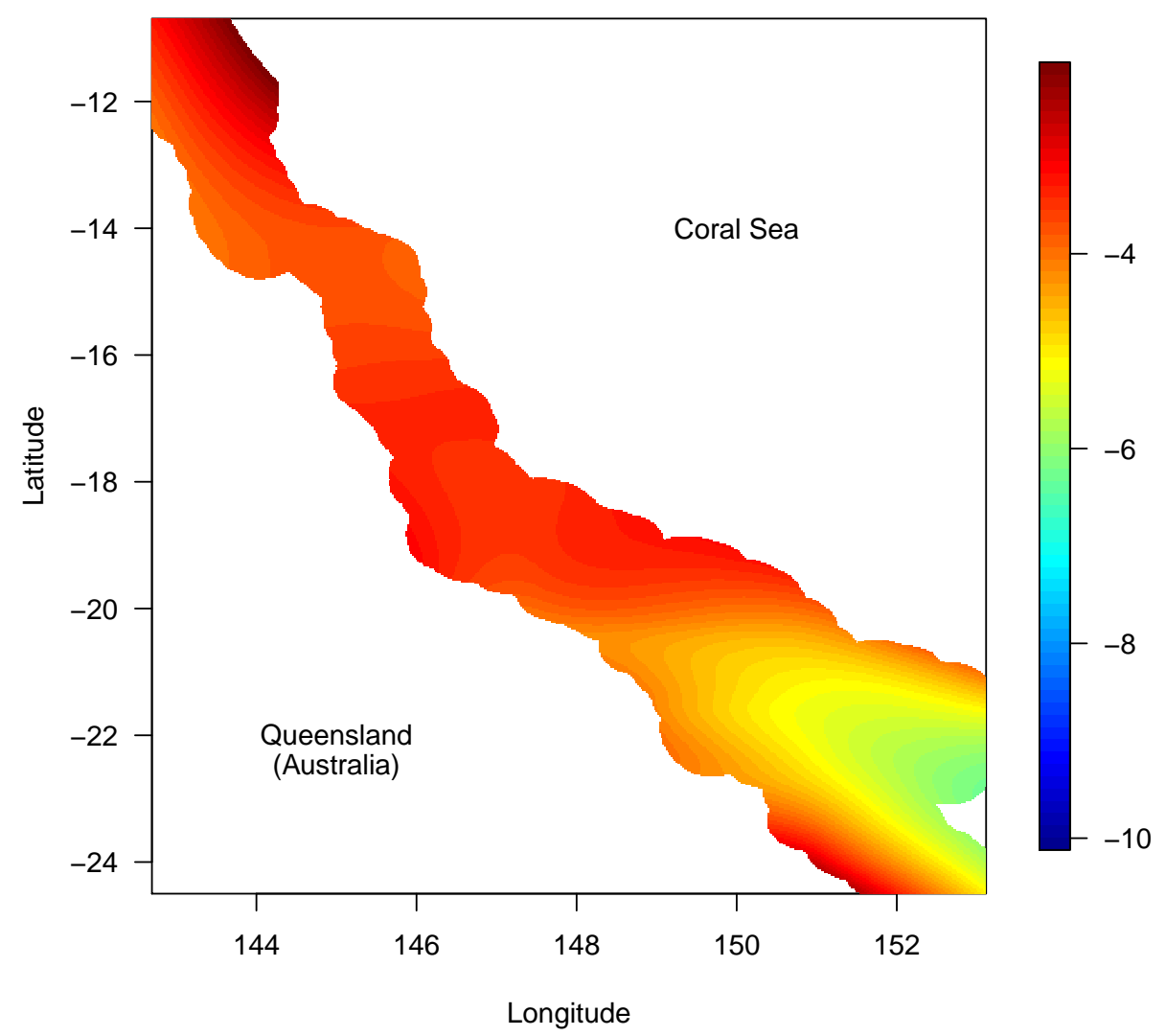

Probability of Presence - Archetype 8 (MIXGL1)

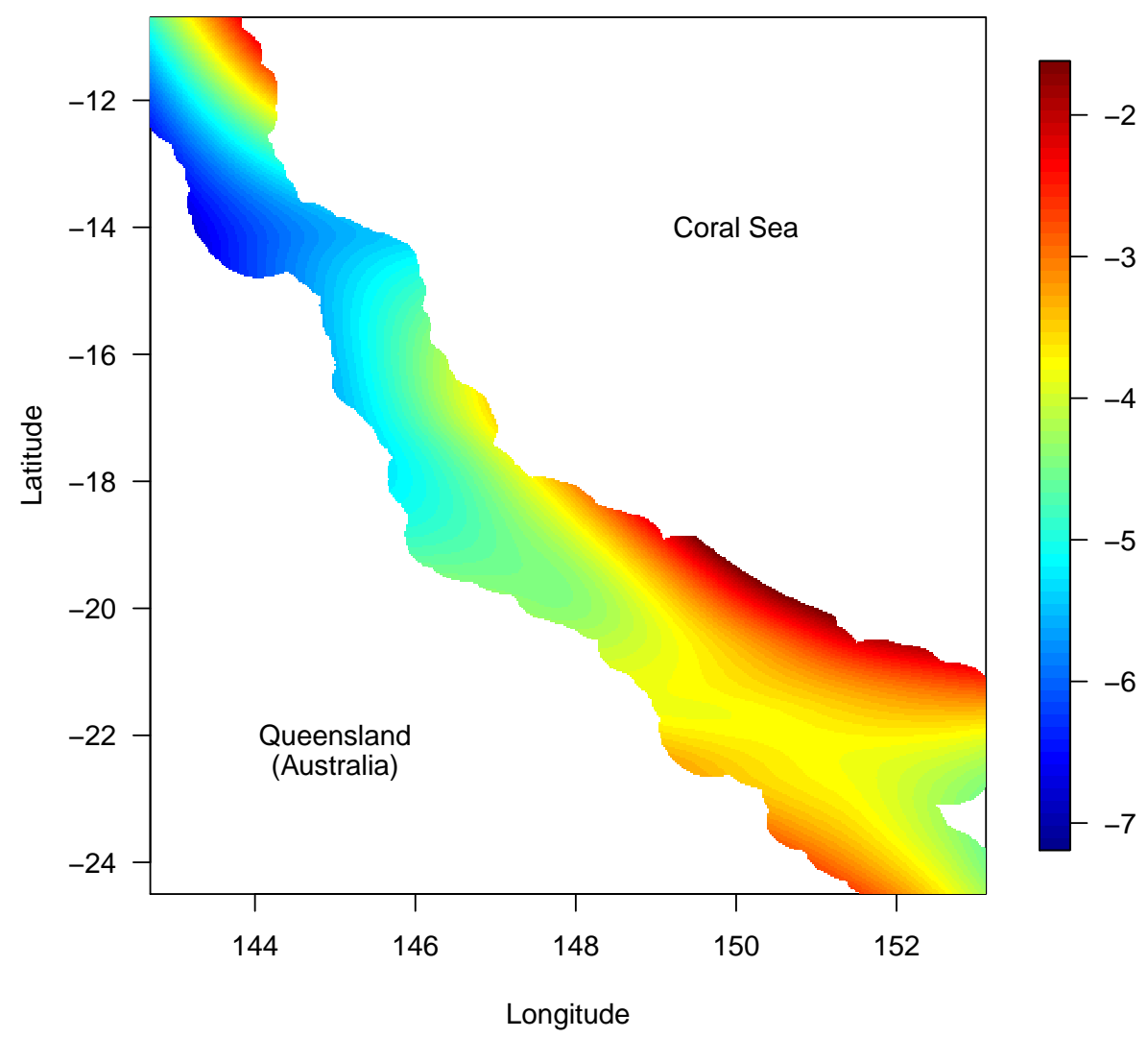


Probability of Presence - Archetype 9 (MIXGL1)

182

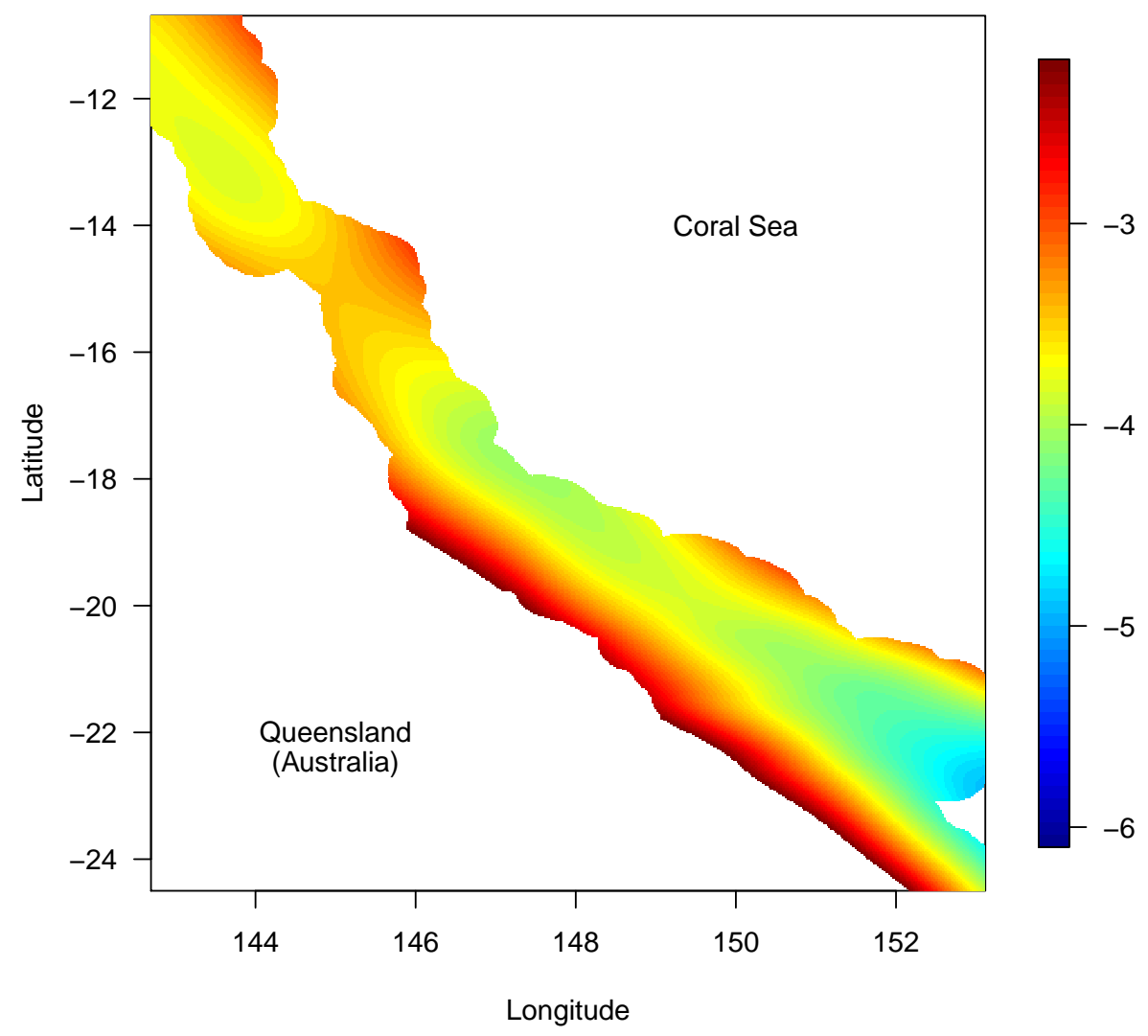

Probability of Presence - Archetype 10 (MIXGL1)

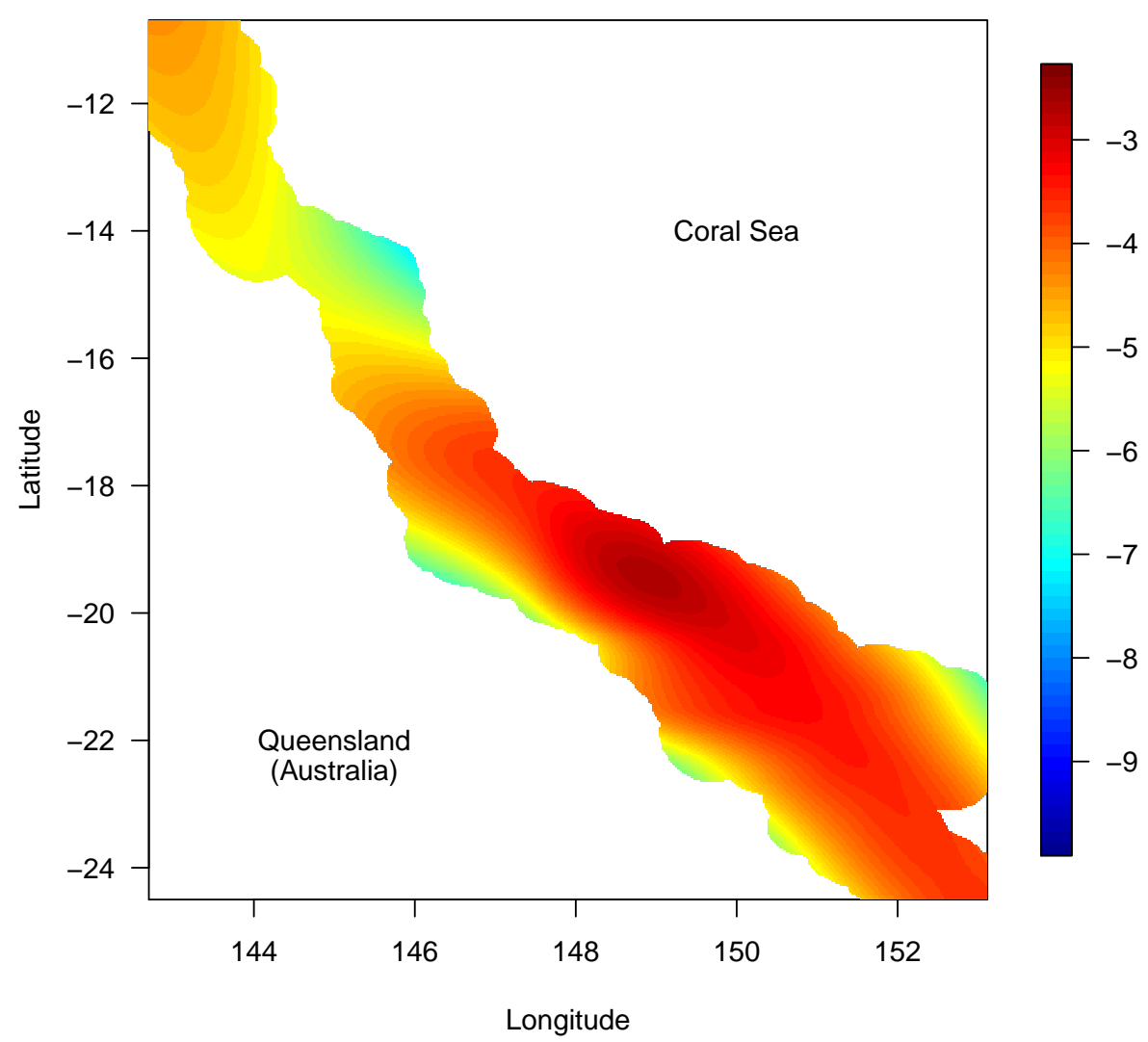


TABle 1

Coefficients for fitted SAM with MIXGL2 penalty. Each column corresponds to an archetype (mixture component). Blank entries represent coefficients which have been shrunk to 0.

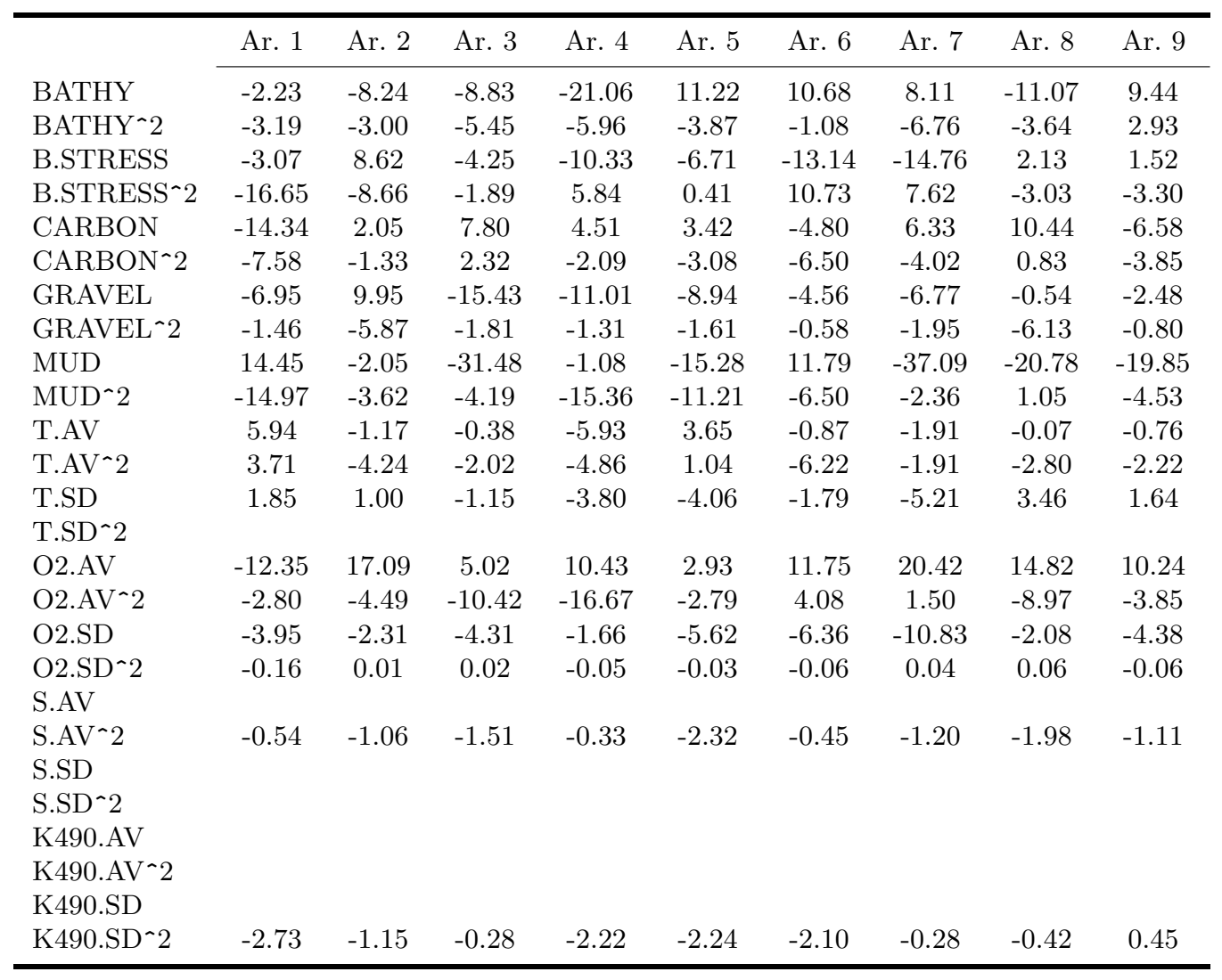


TABLE 2

Coefficients for fitted SAM with MIXGL1 penalty. Each column corresponds to an archetype (mixture component). Blank entries represent coefficients which have been shrunk to 0.

\begin{tabular}{|c|c|c|c|c|c|c|c|c|c|c|}
\hline & Ar. 1 & Ar. 2 & Ar. 3 & Ar. 4 & Ar. 5 & Ar. 6 & Ar. 7 & Ar. 8 & Ar. 9 & Ar. 10 \\
\hline BATHY & -13.14 & 2.19 & -3.33 & -16.70 & 11.04 & 1.83 & 25.67 & -14.82 & 9.38 & -8.52 \\
\hline BATHY^2 $^{\wedge}$ & -2.92 & -4.92 & -8.42 & -8.97 & & -0.94 & -10.91 & -2.75 & & -8.26 \\
\hline B.STRESS & -1.00 & -9.67 & -4.13 & -32.77 & -5.09 & -10.37 & -16.37 & 0.24 & -0.38 & 2.24 \\
\hline B.STRESS^2 & -8.70 & -9.99 & & 17.03 & 1.97 & -11.73 & 9.65 & & -0.55 & -3.28 \\
\hline CARBON & -8.70 & 9.33 & 7.19 & 14.47 & 5.06 & -18.55 & 7.67 & 9.79 & -6.94 & 0.65 \\
\hline $\mathrm{CARBON}^{\wedge} 2$ & -2.62 & 4.48 & 1.94 & -0.90 & -7.09 & -11.07 & -7.87 & & -2.97 & -0.07 \\
\hline GRAVEL & -20.32 & -13.56 & -22.76 & -13.81 & 2.53 & -6.31 & -9.41 & 5.34 & -5.14 & -10.24 \\
\hline GRAVEL`2 & -10.02 & -3.39 & -3.35 & -2.73 & -4.92 & & -5.17 & -7.73 & & -15.69 \\
\hline MUD & -3.10 & -14.95 & -58.10 & -2.50 & -29.58 & 15.28 & -17.93 & -21.84 & -16.70 & -0.94 \\
\hline MUD^2 & -19.97 & -16.34 & -19.41 & -11.98 & & -9.96 & -8.99 & 1.33 & & -5.92 \\
\hline $\begin{array}{l}\text { T.AV } \\
\text { T.AV^2 }\end{array}$ & 0.67 & 9.79 & & -8.85 & -6.66 & 2.20 & & & & -8.82 \\
\hline $\begin{array}{l}\text { T.SD } \\
\text { T.SD^2 }\end{array}$ & & -1.34 & -2.89 & -14.14 & -5.56 & -0.23 & -8.82 & 7.68 & & -1.55 \\
\hline O2.AV & -3.33 & & 4.52 & 2.18 & 10.95 & & 3.00 & 4.65 & 2.55 & 6.90 \\
\hline $\mathrm{O} 2 . \mathrm{AV}^{\wedge} 2$ & & -0.43 & -5.89 & -3.19 & -1.96 & & 0.46 & & & -16.20 \\
\hline $\mathrm{O} 2 . \mathrm{SD}$ & & 0.54 & & -6.70 & -9.83 & -5.08 & -3.85 & & -4.24 & \\
\hline $\mathrm{O} 2 . \mathrm{SD}^{\sim} 2$ & -3.57 & & & -1.15 & & -5.15 & -2.05 & & -1.34 & \\
\hline S.AV & 2.87 & 4.11 & & & & & & & 6.40 & \\
\hline S.AV^2 & -1.15 & -5.79 & -9.57 & & & & & & & -11.04 \\
\hline $\begin{array}{l}\text { S.SD } \\
\text { S.SD^2}\end{array}$ & 3.32 & 5.77 & & & -2.60 & & & & & \\
\hline $\begin{array}{l}\text { K490.AV } \\
\text { K490.AV^2 } \\
\text { K490.SD }\end{array}$ & -0.52 & & & & -28.47 & 0.99 & & & & \\
\hline $\mathrm{K} 490 . \mathrm{SD}^{\sim} 2$ & -8.72 & -7.04 & & & 1.57 & -5.16 & -0.70 & -0.11 & & -9.48 \\
\hline
\end{tabular}

184 Coefficients and Maps for penalized SAM with MIXGL1 penalty. 


\section{APPENDIX D: ADDITIONAL RESULTS FOR SECTION 5}

TABLE 3

Mean sensitivity/specificity for various sample sizes and $\pi_{1}=0.7$. Transitioning from models $I$ to $I V$, the proportion of completely uninformative covariates increases. MIXGL1 performed very well overall, while the specificity of ADL, MIXLASSO- $\ell_{2}$, and MIXSCAD- $\ell_{2}$ drop a small but noticeable amount as the number of complete uninformative covariates increases. As expected, MIXGL2 is the best performer in Model IV.

\begin{tabular}{ccccccc}
\hline \multirow{2}{*}{$n$} & & \multicolumn{5}{c}{ Sensitivity/Specificity } \\
& Model & MIXGL1 & MIXGL2 & ADL & MIXLASSO- $\ell_{2}$ & MIXSCAD- $\ell_{2}$ \\
\cline { 3 - 7 } 100 & I & $0.933 / 0.942$ & $0.935 / 0.108$ & $0.947 / 0.870$ & $0.983 / 0.183$ & $0.950 / 0.703$ \\
& II & $0.924 / 0.957$ & $0.920 / 0.430$ & $0.944 / 0.867$ & $0.981 / 0.152$ & $0.935 / 0.708$ \\
& III & $0.918 / 0.955$ & $0.913 / 0.772$ & 0.9270 .845 & $0.965 / 0.238$ & $0.922 / 0.697$ \\
& IV & $0.921 / 0.967$ & $1 / 0.970$ & $0.937 / 0.835$ & $0.956 / 0.195$ & $0.938 / 0.703$ \\
& & & & & & \\
200 & I & $0.957 / 0.973$ & $0.897 / 0.462$ & $0.965 / 0.929$ & $0.982 / 0.413$ & $0.962 / 0.852$ \\
& II & $0.965 / 0.982$ & $0.918 / 0.682$ & $0.970 / 0.929$ & $0.983 / 0.451$ & $0.972 / 0.823$ \\
& III & $0.966 / 0.989$ & $0.919 / 0.882$ & $0.976 / 0.904$ & $0.992 / 0.396$ & $0.968 / 0.820$ \\
& IV & $0.988 / 0.982$ & $1 / 0.993$ & $0.977 / 0.888$ & $0.991 / 0.381$ & $0.974 / 0.817$ \\
& & & & & & \\
400 & I & $0.986 / 0.988$ & $0.949 / 0.654$ & $0.997 / 0.949$ & $0.997 / 0.579$ & $0.996 / 0.906$ \\
& II & $0.991 / 0.990$ & $0.938 / 0.789$ & $0.992 / 0.958$ & $0.995 / 0.593$ & $0.990 / 0.911$ \\
& III & $0.985 / 0.994$ & $0.945 / 0.907$ & $0.991 / 0.954$ & $0.994 / 0.533$ & $0.983 / 0.891$ \\
& IV & $0.991 / 0.990$ & $1 / 1$ & $0.994 / 0.954$ & $0.999 / 0.578$ & $0.995 / 0.876$ \\
\hline
\end{tabular}


FIG 1. Predictive log-likelihood (centered) as a function of simulation model for $\pi_{1}=0.7$. The

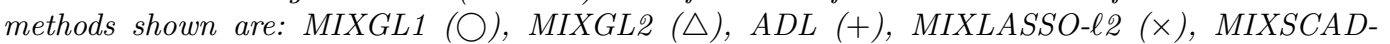
ใ2 $(\diamond)$. As we move from models I to $I V$, the proportion of completely uninformative covariates increases. MIXGL1 predicted best overall, with the predictive performance of MIXGL2 improving dramatically as we transition from models I to IV.
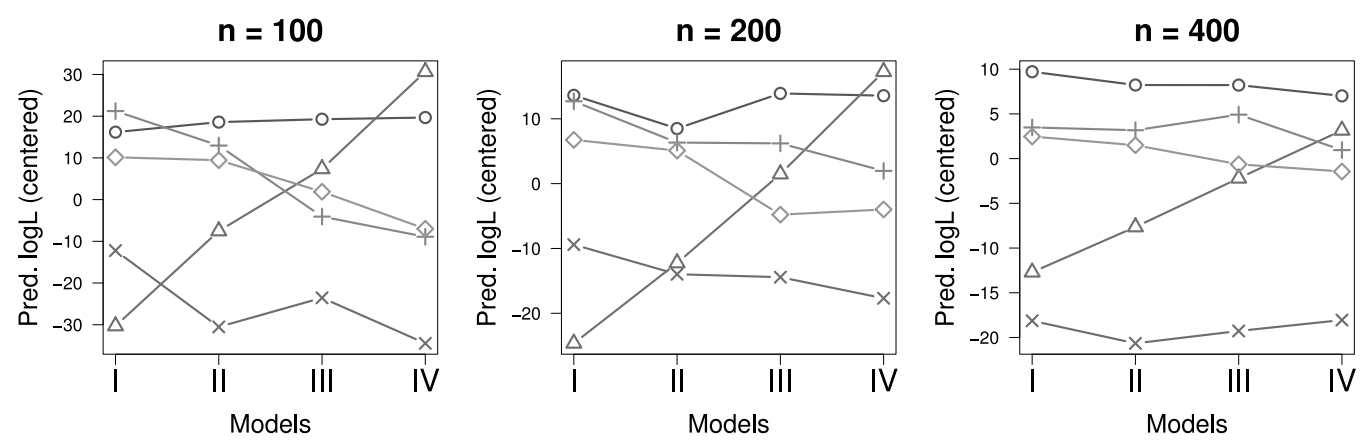

APPENDIX E: ADDITIONAL SIMULATION STUDY

We performed a second simulation with finite mixtures of linear regressions, i.e. normal response, and $K=4$ components. The sample size was fixed $n=400$, with the number of covariates determined by $p_{n}=\left\lceil 4 n^{1 / 4}\right\rceil$, leading to 18 predictors. Covariates $\boldsymbol{x}_{i} ; i=1, \ldots, n$ were generated from a standard multivariate Gaussian distribution with AR1 correlation structure $\operatorname{Cor}\left(x_{i r}, x_{i s}\right)=0.5^{|r-s|}$. Responses were then simulated from a four component, homoscedastic normal FMR model with $\sigma^{2}=1$ and the four models below. Performance was assessed using mean sensitivity (proportion of true non-zeros estimated to be non-zero) and sensitivity (proportion of true zeros estimated to be zero). As before, to deal with the problem of label-switching prior to calculating sensitivity and specificity, we permuted the estimated coefficients so as to minimize the $\ell_{2}$-norm between the estimated and true coefficients. 


$$
\begin{aligned}
& \left(\beta_{01}, \boldsymbol{\beta}_{1}\right)=\left(\begin{array}{lllllllllll}
8, & 4, & 3, & 2, & 0, & 0,0,0, & 0, & 0, & 0, & 0, & 0,
\end{array}\right)
\end{aligned}
$$

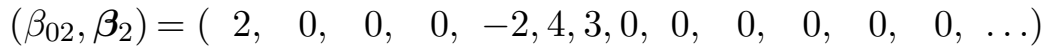

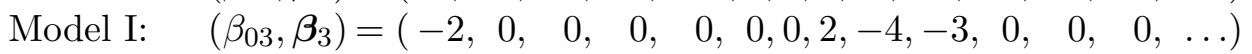

$$
\begin{aligned}
& \left(\beta_{04}, \boldsymbol{\beta}_{4}\right)=(-8,0,0, \quad 0,0,0,0,0,0,0,-2,-3,-4, \ldots) \\
& \left(\beta_{02}, \boldsymbol{\beta}_{2}\right)=(2,-2,4, \quad 3, \quad 0,0,0,0,0, \quad 0, \quad 0, \quad 0, \quad 0, \ldots) \\
& \text { Model II: } \quad\left(\beta_{03}, \boldsymbol{\beta}_{3}\right)=\left(\begin{array}{llll}
-2,0, & 0,0,0,0,0,2,-4,-3,0, & 0, & 0, \ldots)
\end{array}\right. \\
& \left(\beta_{04}, \boldsymbol{\beta}_{4}\right)=(-8,0,0,0,0,0,0,0,0,0,-2,-3,-4, \ldots) \\
& \left(\beta_{02}, \boldsymbol{\beta}_{2}\right)=(2,-2,4,3,0,0,0,0,0,0,0,0,0, \ldots) \\
& \text { Model III: } \quad\left(\beta_{03}, \boldsymbol{\beta}_{3}\right)=(-2,2,-4,-3,0,0,0,0,0, \quad 0,0,0,0, \ldots) \\
& \left(\beta_{04}, \boldsymbol{\beta}_{4}\right)=(-8,0, \quad 0,0, \quad 0,0,0,0,0,0,-2,-3,-4, \ldots)
\end{aligned}
$$

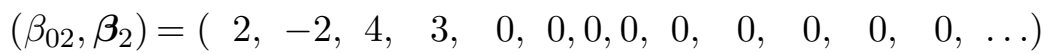

$$
\begin{aligned}
& \text { Model IV: } \quad\left(\beta_{03}, \boldsymbol{\beta}_{3}\right)=(-2,2,-4,-3,0,0,0,0,0,0,0,0,0, \ldots) \\
& \left(\beta_{04}, \boldsymbol{\beta}_{4}\right)=(-8,-2,-3,-4,0,0,0,0,0,0,0,0,0, \ldots)
\end{aligned}
$$

Two configurations of mixing proportions were considered, $\boldsymbol{\pi}^{0}=(0.25,0.25,0.25,0.25)$ and $\boldsymbol{\pi}^{0}=(0.15,0.4,0.25,0.2) .100$ datasets were simulated for each combination of model type (I,II,III,IV) and $\boldsymbol{\pi}^{0}$, with $K=4$ assumed to be known in advance.

Given the increased number of components and covariates in this setting, it was not surprising that both sensitivity and specificity values were lower than those seen in $K=2$ components simulation study in the main manuscript. On the other hand, all methods actually improved in performance when the proportion of complete uninformative covariates was increased from models I to IV (Table 4). MIXGL1 was again the best performer - it has significantly higher specificity than the three methods which did not penalized on a per-covariate basis, with no appreciable loss in sensitivity. As expected, MIXGL2 improved dramatically when moving from models I and IV. The two penalties which were applied on a per-component overfitted the most, particularly MIXLASSO- $\ell_{2}$. The performance of all methods worsened slightly with uneven mixing proportions, although the same trends can be observed as in the balanced case.

The predictive performance of all methods actually worsened as we transitioned from models I to IV, with the exception of MIXGL2 which dramatically improved. As in $K=2$ components simulation, MIXGL1 predicted best in both the balanced and unbalanced mixing proportions case, and regardless of the proportion of completely uninformative covariates in the simulation model. 
TABLE 4

Mean sensitivity/specificity for additional simulation study and two configurations of $\boldsymbol{\pi}^{0}$.

\begin{tabular}{cccccc}
\hline \multirow{2}{*}{ Model } & MIXGL1 & MIXGL2 & ADL & MIXLASSO- $\ell_{2}$ & MIXSCAD- $\ell_{2}$ \\
\cline { 2 - 6 } & \multicolumn{5}{c}{$\pi^{0}=(0.25,0.25,0.25,0.25)$} \\
I & $0.797 / 0.920$ & $0.961 / 0.377$ & $0.799 / 0.863$ & $0.876 / 0.721$ & $0.812 / 0.807$ \\
II & $0.894 / 0.950$ & $0.988 / 0.574$ & $0.895 / 0.887$ & $0.897 / 0.727$ & $0.891 / 0.840$ \\
III & $0.936 / 0.960$ & $0.998 / 0.785$ & $0.917 / 0.892$ & $0.892 / 0.772$ & $0.892 / 0.854$ \\
IV & $0.998 / 0.989$ & $1 / 0.998$ & $0.995 / 0.924$ & $0.964 / 0.797$ & $0.946 / 0.878$ \\
& & \multicolumn{4}{c}{$\pi^{0}=(0.15,0.4,0.25,0.2)$} \\
I & $0.785 / 0.912$ & $0.935 / 0.379$ & $0.789 / 0.844$ & $0.841 / 0.685$ & $0.790 / 0.811$ \\
II & $0.881 / 0.933$ & $0.974 / 0.537$ & $0.861 / 0.846$ & $0.897 / 0.669$ & $0.858 / 0.791$ \\
III & $0.917 / 0.949$ & $0.994 / 0.712$ & $0.922 / 0.858$ & $0.929 / 0.688$ & $0.912 / 0.845$ \\
IV & $0.985 / 0.982$ & $1 / 0.976$ & $0.990 / 0.905$ & $0.976 / 0.751$ & $0.953 / 0.857$ \\
\hline
\end{tabular}

FIG 2. Predictive log-likelihood (centered) as a function of simulation model for $\boldsymbol{\pi}^{0}=$ $(0.25,0.25,0.25,0.25)$ (left) and $\boldsymbol{\pi}^{0}=(0.15,0.4,0.25,0.2)$ (right). The methods shown are: MIXGL1

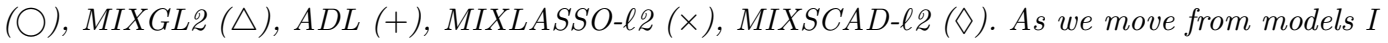
to $I V$, the proportion of completely uninformative covariates increases.
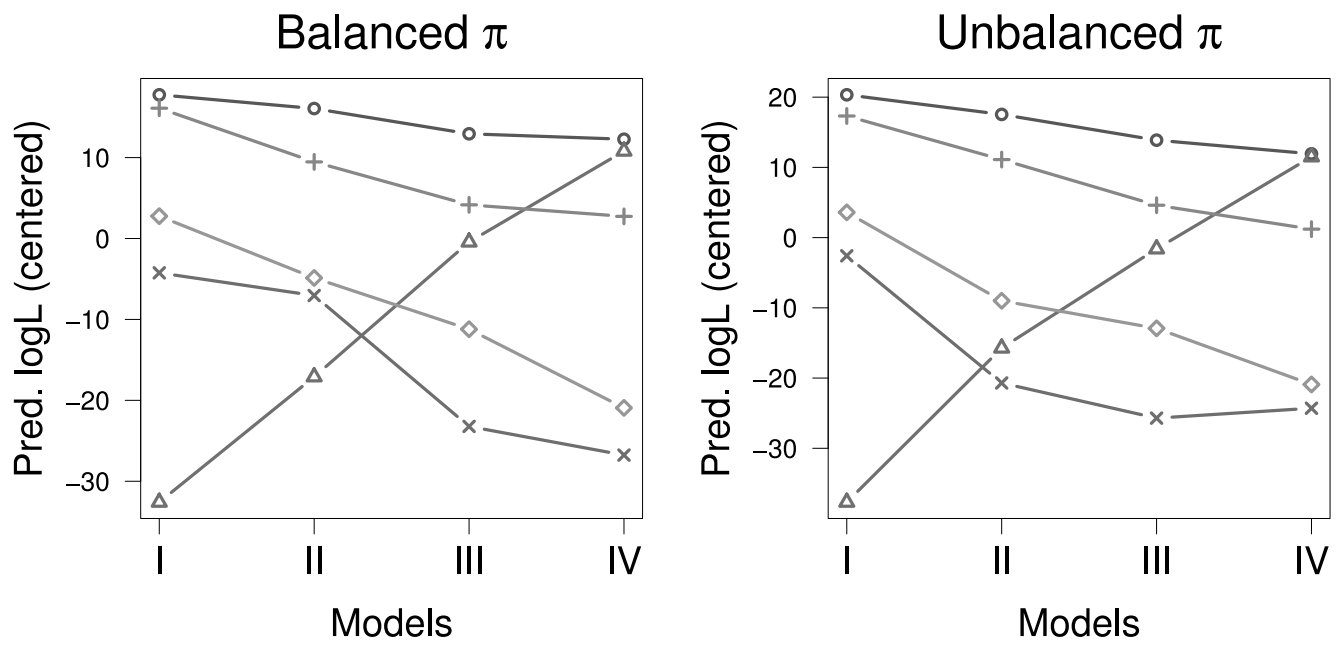


\section{REFERENCES}

Dempster, A. P., Laird, N. M., and Rubin, D. B. (1977). Maximum likelihood from incomplete data via the EM algorithm. Journal of the Royal Statistical Society: Series B (Methodological), 39:1-38.

Fan, J. and Li, R. (2001). Variable selection via nonconcave penalized likelihood and its oracle properties. Journal of the American Statistical Association, 96:1348-1360.

Fan, J. and Peng, H. (2004). Nonconcave penalized likelihood with a diverging number of parameters. Annals of Statistics, 32:928-961.

Hunter, D. R. and Li, R. (2005). Variable selection using MM algorithms. The Annals of statistics, $33: 1617-1642$.

Meng, X.-L. and Rubin, D. B. (1993). Maximum likelihood estimation via the ECM algorithm: A general framework. Biometrika, 80:267-278.

Nelder, J. and Mead, R. (1965). A simplex algorithm for function minimization. Computer Journal, $7: 308-313$.

Wikipedia (2015). Expectationmaximization algorithm — wikipedia, the free encyclopedia. [Online; accessed 7-February-2015].

Zhou, N. and Zhu, J. (2007). Group variable selection via a hierarchical Lasso and its oracle property. Technical Report 464, University of Michigan. 